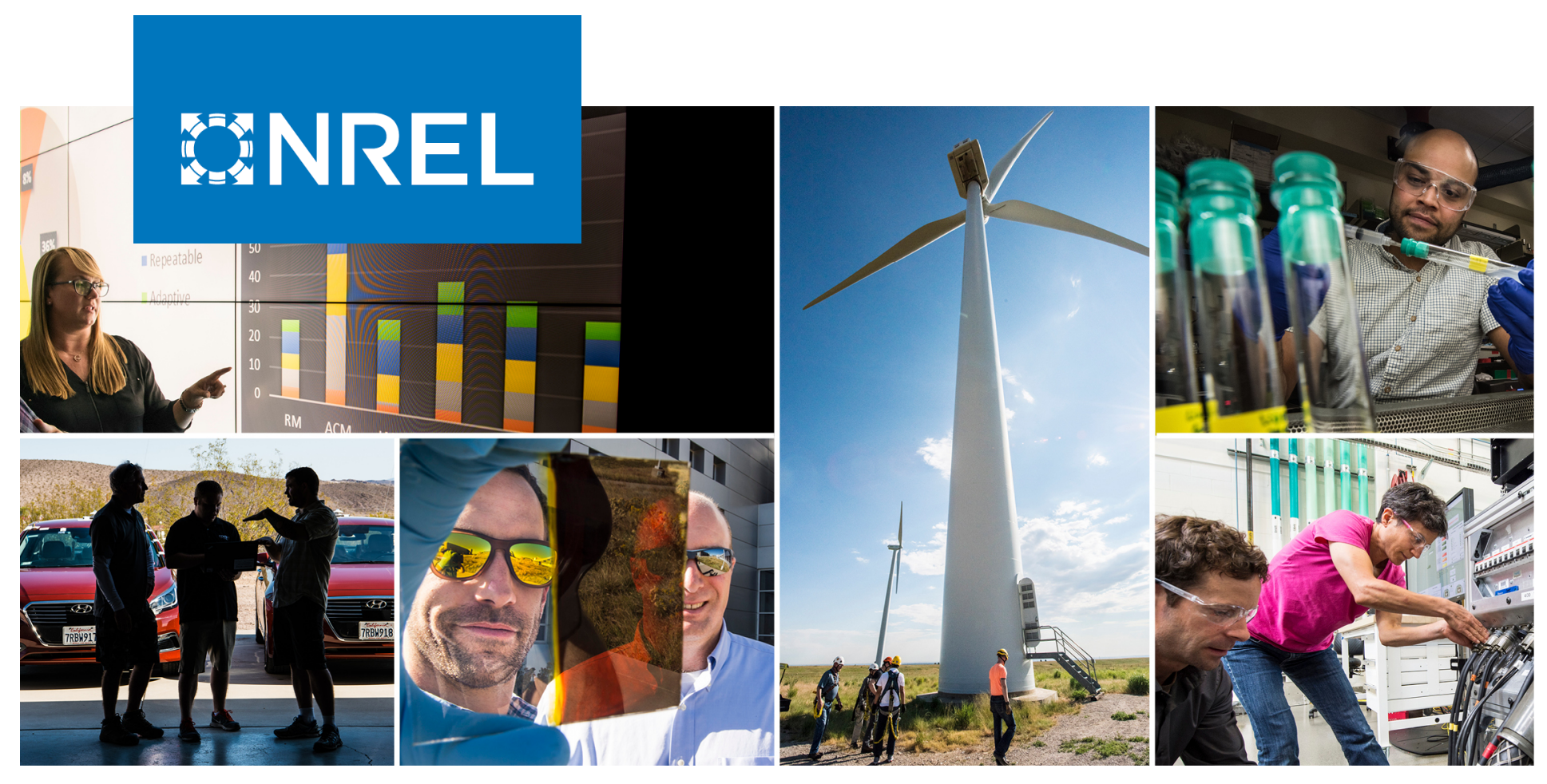

\title{
Emergency Diesel Generator Reliability and Installation Energy Security
}

Jeffrey Marqusee, Sean Ericson, and Don Jenket

National Renewable Energy Laboratory

NREL is a national laboratory of the U.S. Department of Energy

Office of Energy Efficiency \& Renewable Energy

Operated by the Alliance for Sustainable Energy, LLC

This report is available at no cost from the National Renewable Energy Laboratory (NREL) at www.nrel.gov/publications.

\section{Technical Report}

NREL/TP-5C00-76553

April 2020 


\section{GNREL}

\section{Emergency Diesel Generator Reliability and Installation Energy Security}

Jeffrey Marqusee, Sean Ericson, and Don Jenket

National Renewable Energy Laboratory

\section{Suggested Citation}

Marqusee, Jeffrey, Sean Ericson, and Don Jenket. 2020. Emergency Diesel Generator Reliability and Installation Energy Security. Golden, CO: National Renewable Energy Laboratory. NREL/TP-5C00-76553. https://www.nrel.gov/docs/fy20osti/76553.pdf.

NREL is a national laboratory of the U.S. Department of Energy Office of Energy Efficiency \& Renewable Energy Operated by the Alliance for Sustainable Energy, LLC

This report is available at no cost from the National Renewable Energy Laboratory (NREL) at www.nrel.gov/publications.

Contract No. DE-AC36-08GO28308
Technical Report NREL/TP-5C00-76553 April 2020

National Renewable Energy Laboratory 15013 Denver West Parkway Golden, CO 80401 303-275-3000 • www.nrel.gov 


\section{NOTICE}

This work was authored by the National Renewable Energy Laboratory, operated by Alliance for Sustainable Energy, LLC, for the U.S. Department of Energy (DOE) under Contract No. DE-AC36-08GO28308. Support for the work was also provided by the Department of Defense's Environmental Security Technology Certification Program (ESTCP) under Agreement IAG-18-02080. The views expressed herein do not necessarily represent the views of the DOE or the U.S. Government.

This report is available at no cost from the National Renewable Energy Laboratory (NREL) at www.nrel.gov/publications.

U.S. Department of Energy (DOE) reports produced after 1991 and a growing number of pre-1991 documents are available free via www.OSTI.gov.

Cover Photos by Dennis Schroeder: (clockwise, left to right) NREL 51934, NREL 45897, NREL 42160, NREL 45891, NREL 48097, NREL 46526.

NREL prints on paper that contains recycled content. 


\section{List of Acronyms}

DER

DoD

EDG

FTS

IEEE

MTBF

MTTF

MTTM

MTTR

NRC

NREL

OA

PREP

SCADA distributed energy resource

U.S. Department of Defense

emergency diesel generator

failure to start

Institute of Electrical and Electronics Engineers

mean time between failure

mean time to failure

mean time to maintain

mean time to repair

Nuclear Regulatory Commission

National Renewable Energy Laboratory operational availability

U.S. Army Corps of Engineers Power Reliability Enhancement Program

Supervisory Control and Data Acquisition 


\section{Executive Summary}

The U.S. Department of Defense's (DoD's) fixed installations — or military bases — are a critical element of national security. Military bases support the maintenance and deployment of weapons systems, training and mobilization of combat forces, and provide direct support to combat operations. They also play a critical role in homeland defense and during domestic emergencies by providing support to civil authorities. Fixed installations do not operate without energy and they rely largely on electricity to support critical missions and functions. Installations are dependent on a commercial grid that is vulnerable to disruption due to severe weather, physical attacks, and cyberattacks.

The current default solution for backup energy at military installations relies on emergency diesel generators (EDGs). This is most often accomplished by either a single stand-alone generator or two generators tied to an individual building with critical loads. Less commonly, but with increasing frequency, diesel generators are networked and serve as the primary distributed energy resource for a microgrid. EDGs can fail more often than recognized and their reliability must be considered when evaluating energy backup system architectures. This report provides an analytic approach to quantitively assess the impact of an EDG's reliability on both stand-alone building tied systems and microgrids.

Based on a new analysis of existing empirical data, Figure ES- 1 shows the reliability of an EDG as a function of outage duration and level maintenance. A well-maintained EDG is one that rigorously follows Unified Facility Criteria guidance (UFC 3-540-07). A poorly maintained EDG is unlikely to provide power for durations longer than a few days and has a reliability of only $80 \%$ at 12 hours. This figure reinforces the importance of following the current guidance on EDG maintenance. But even wellmaintained EDGs have a reliability of only $80 \%$ at two weeks. Thus, a single well-maintained EDG cannot guarantee emergency power for critical loads over multiday outages.

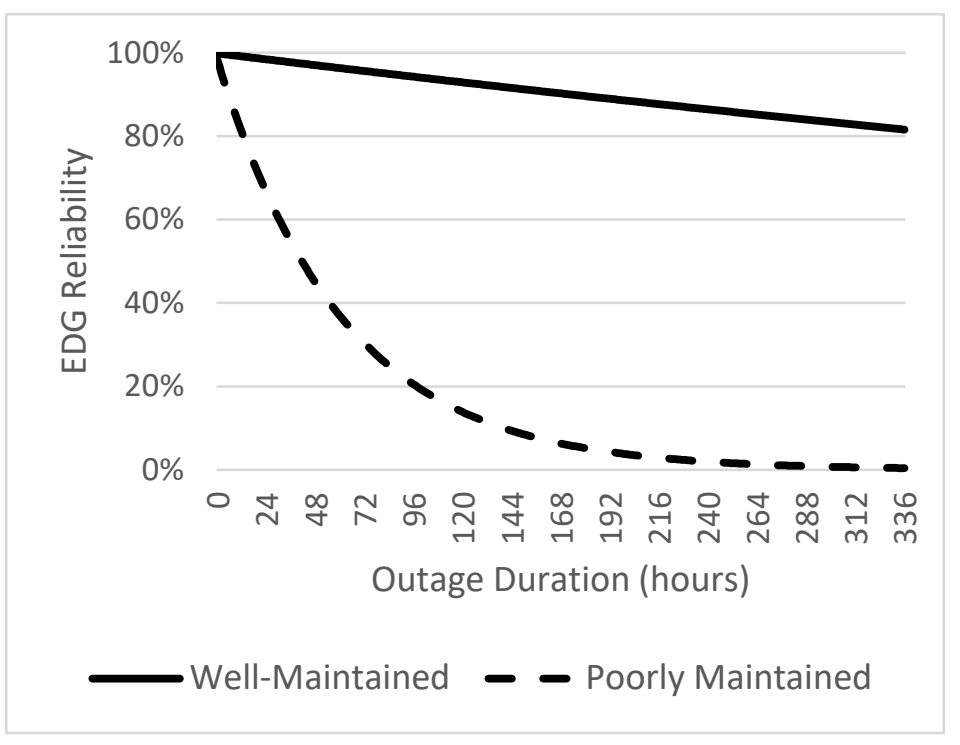

Figure ES-1. The reliability of a single EDG over two weeks (336 hours)

Calculations of the reliability of different backup energy architectures for four model installations are provided (see Table ES- 1). The installation energy scenarios include a range of critical load sizes typically found on military installations and realistic hourly load profiles. The scenarios include outages ranging from one hour to two weeks and cover typical conditions found on small national guard and reserve bases up to very large domestic active military installations. These scenarios can serve as screening level benchmarks for the expected performance on fixed 
installations worldwide. The tool to assess backup power system reliability is available, through the National Renewable Energy Laboratory (NREL), for site-specific assessments to evaluate current energy assurance performance and potential future alternative systems.

Table ES- 1. Annual Hourly Peak Critical Load, Number of Critical Buildings for the Small, Medium, Large, and Very Large Bases Sizes Modeled

\begin{tabular}{|l|l|l|l|l|}
\hline Base & Small & Medium & Large & Very Large \\
\hline Peak Annual Critical Load (MW) & 1 & 5 & 10 & 20 \\
\hline \# Buildings With Critical Loads & 8 & 40 & 80 & 160 \\
\hline
\end{tabular}

Three base level reliability metrics (probability of supporting 100\% of critical load, fraction of lost load, and probability to satisfy the highest priority critical loads) are examined for wellmaintained EDGs. Poorly maintained generators do not meet the needs of military installations independent of how they are arranged. Even in a microgrid configuration, the loss of multiple generators within a few days due to poor maintenance yields inadequate performance.

The probability that all critical load will be $100 \%$ supported as a function of outage duration up to two weeks is shown below for the small and very large base. The performance of two systems are shown; a microgrid with $\mathrm{N}+1$ back-up generators (referred to as an $\mathrm{N}+1$ microgrid) where $\mathrm{N}$ generators are needed to satisfy the annual peak critical load, and a system where one EDG is tied to each building.
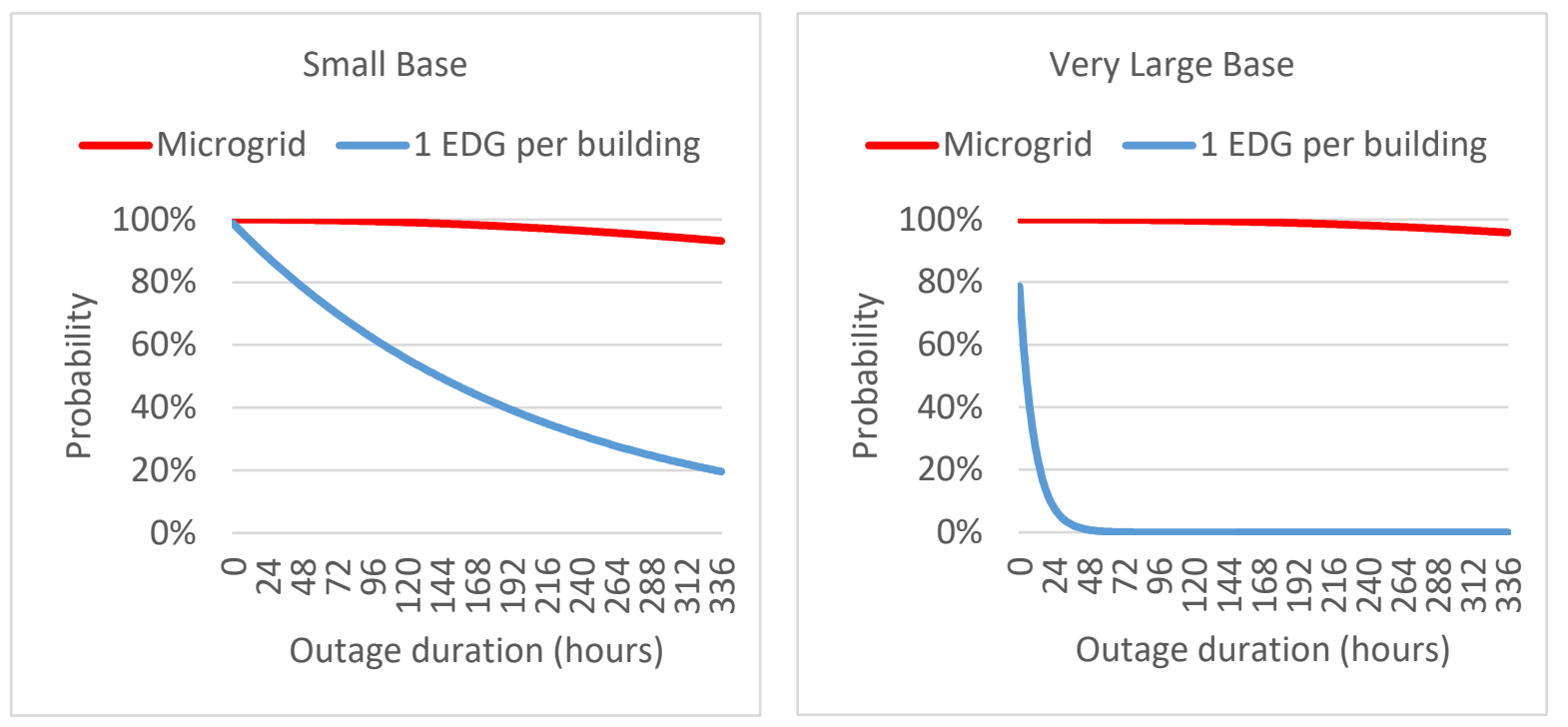

Figure ES- 2. The probability of an N+1 microgrid and a single EDG per building architecture meeting $100 \%$ of the critical load requirement for outages up to 14 days (336 hours). For small (left) and very large (right) bases.

Due to the ability of EDGs to share load in a microgrid, this architecture maintains a high probability of meeting a $100 \%$ of the critical load for two weeks for all bases. Stand-alone generators have a small probability of providing power for all buildings with a critical load for a multiday outage. 
Table ES- 2 shows the $90 \%$ confidence intervals for the fraction of lost load for the $\mathrm{N}+1$ microgrid configuration and the fraction of buildings without power for a single stand-alone EDG per building for outages of 7 and 14 days for all four bases. The fraction of buildings without power in a stand-alone system is independent of the size of the base.

Table ES- 2. $90 \%$ Confidence Ranges for the Fraction of Load a Microgrid Must Shed and the Fraction of Buildings with Critical Load That Will Not Have Power if One Uses a Single StandAlone Building-Tied EDG at 7 and 14 Days

\begin{tabular}{|c|c|c|c|c|c|}
\hline Architecture & Microgrid & Microgrid & Microgrid & Microgrid & Stand-Alone \\
\hline Bases & Small & Medium & Large & Very Large & All \\
\hline 7 days & $0.1 \%-0.7 \%$ & $0.0 \%-0.2 \%$ & $0.0 \%-0.2 \%$ & $0.0 \%-0.2 \%$ & $7 \%-13 \%$ \\
\hline 14 days & $0.7 \%-3.4 \%$ & $0.2 \%-1.5 \%$ & $0.2 \%-1.5 \%$ & $0.2 \%-1.7 \%$ & $13 \%-25 \%$ \\
\hline
\end{tabular}

In the microgrid case, the loss of generation can be managed by shedding lower priority critical loads to maintain the microgrid's stability. In the case of building-tied systems, no action can compensate for the EDGs' failures.

Finally, we look at the impact on the highest priority critical loads, typically only a fraction of the total critical load. These are loads that are required to support high priority critical missions that must be sustained. For this case, we will compare an $\mathrm{N}+1$ microgrid architecture to two EDGs per building. Figure ES- 3 below shows the probability of meeting the highest priority load for situations where the high priority load is $25 \%$ of the total critical load for a microgrid and for the two stand-alone EDGs per building. The microgrid essentially has a $100 \%$ probability because it can prioritize which loads are the most important and preferentially send power to those loads. Stand-alone building-tied systems, even when two EDGs are tied to each building, cannot provide high confidence that the highest priority loads will be supported. 


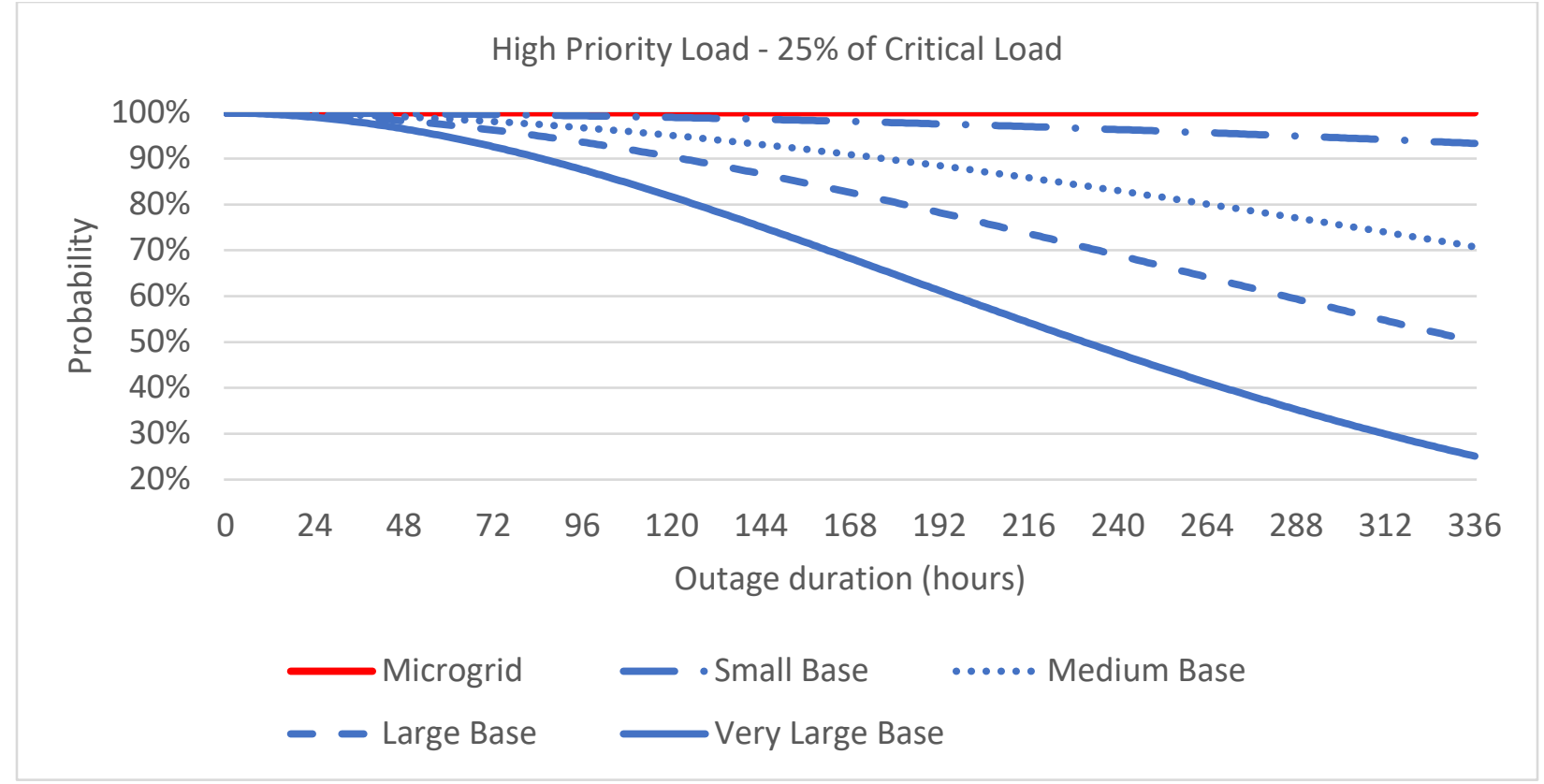

Figure ES- 3 . Probability of meeting $25 \%$ of highest priority critical load for small, medium, large, and very large bases. Building-tied systems are shown in blue and microgrids are shown in red. Building-tied systems have two EDGs per building and microgrids for all size bases overlap.

All three metrics provide overwhelming evidence that stand-alone building-tied EDG systems, even when two EDGs are used, cannot provide the level of confidence required by DoD installations for power to be available to support critical missions during a multiday grid outage. Diesel generator based microgrid configurations provide a robust source of power for critical loads due to their network configuration and ability to share load. But microgrid architectures do introduce other vulnerabilities that must be managed, including cyber vulnerabilities and dependence on the on-base distribution system.

Emergency diesel generators must be wellmaintained if they are to be relied on for providing power longer than a few hours. If backup power is required for multiple days, stand-alone building-tied emergency diesel generators cannot be relied on by themselves to provide backup power for critical loads, and a microgrid should be considered. 


\section{Table of Contents}

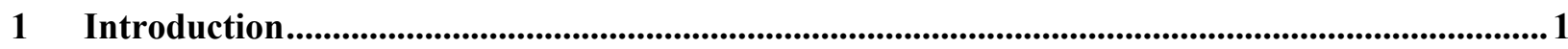

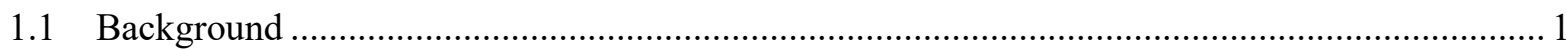

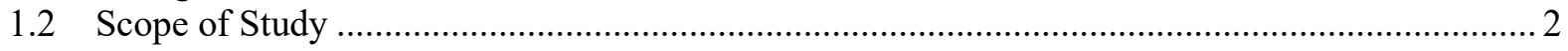

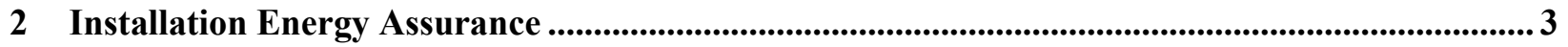

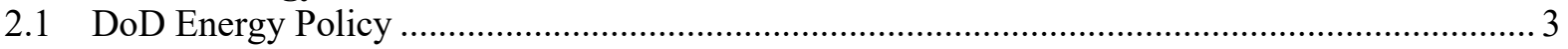

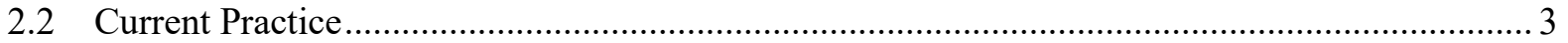

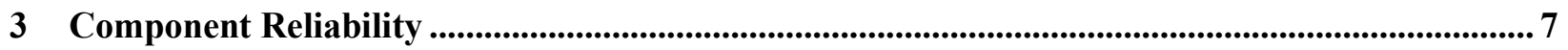

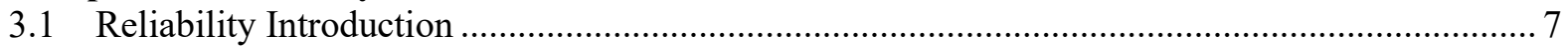

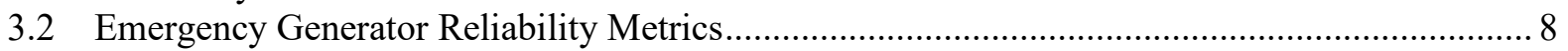

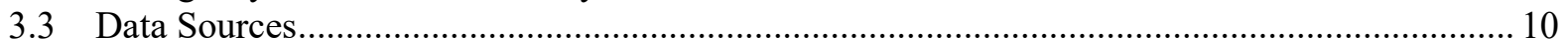

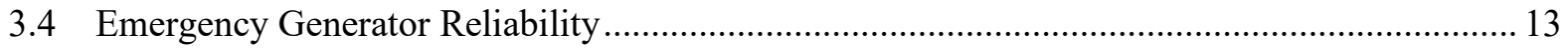

4 System Reliability ................................................................................................................................ 18

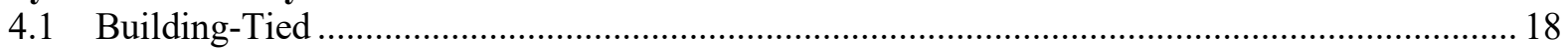

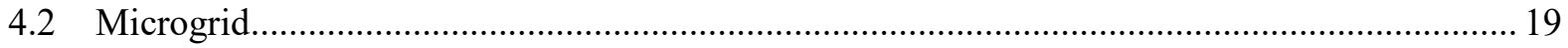

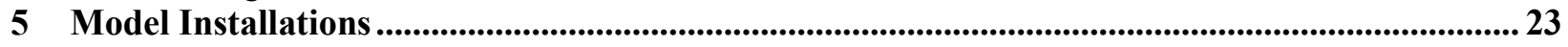

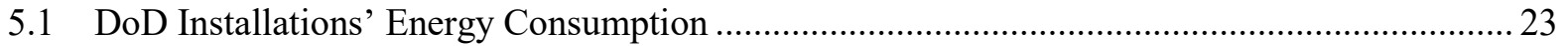

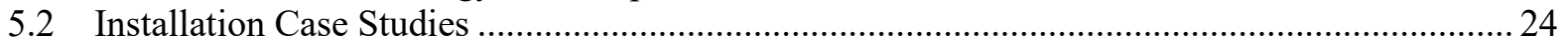

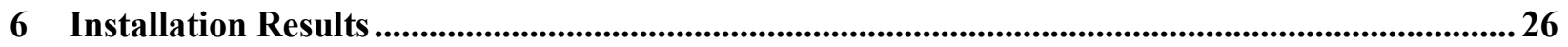

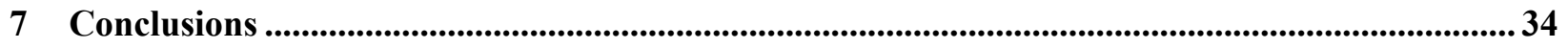

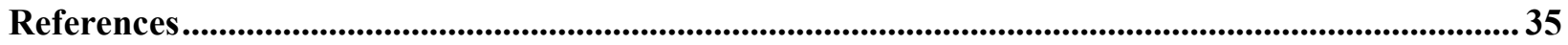




\section{List of Figures}

Figure ES- 1. The reliability of a single EDG over two weeks (336 hours) .............................................

Figure ES- 2 . The probability of an $\mathrm{N}+1$ microgrid and a single EDG per building architecture meeting $100 \%$ of the critical load requirement for outages up to 14 days (336 hours). For small (left) and very large (right) bases.................................................................................................... vi

Figure ES- 3. Probability of meeting $25 \%$ of highest priority critical load for small, medium, large,

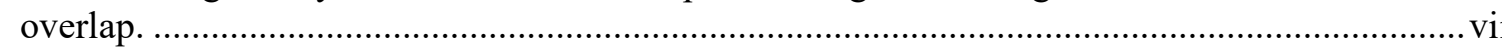

\section{and very large bases. Building-tied systems are shown in blue and microgrids are shown in
red. Building-tied systems have two EDGs per building and microgrids for all size bases \\ and very large bases. Building-tied systems are shown in blue and microgrids are shown
red. Building-tied systems have two EDGs per building and microgrids for all size bases}

Figure 1. Historical approach—stand-alone generators tied to individual buildings............................... 3

Figure 2. Microgrid with larger networked generators ........................................................................ 4

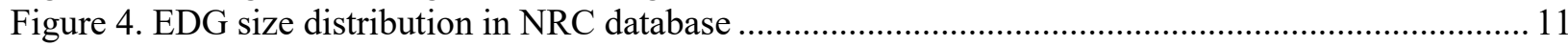

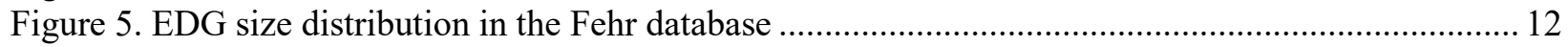

Figure 6. Distribution of repair times, including logistics for EDGs after a failure ................................ 14

Figure 7. The reliability of a single EDG over two weeks (336 hours) ................................................. 16

Figure 8 . The reliability of a single EDG for outages less than half a day (12 hours)............................. 16

Figure 9. Mean and 90\% confidence interval reliabilities for a well-maintained EDG for outages up to

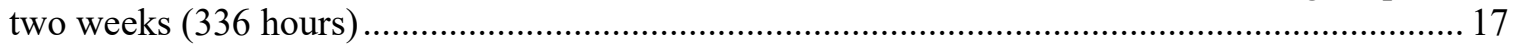

Figure 10. Critical load profile for large base in units of kWh and EDG redundancy ............................ 19

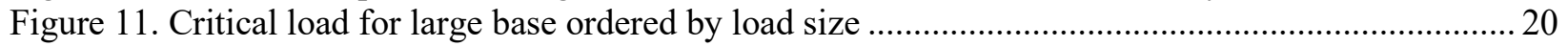

Figure 12. Peak hourly critical load for all DoD installation with buildings with more than 1 million

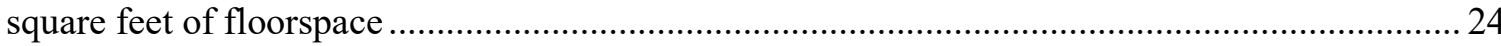

Figure 13. Modeled base critical load profiles with generator step sizes for small (top left), medium (top right), large (bottom left), and very large (bottom right) bases ............................................ 25

Figure 14. The probability of meeting $100 \%$ of the critical load requirement for outages up to 14 days (336 hours). Small (top left), medium (top right), large (bottom left), and very large (bottom right) bases.

Figure 15. Mean expected lost load for a single EDG per building architecture (blue) and microgrids (red) on different size bases.

Figure 16. Probability of meeting $10 \%$ and $25 \%$ of highest priority critical load for small, medium, large, and very large bases. The red curve represents the results for all bases supported by a microgrid. The blue results are for bases where two EDGs are deployed for every building containing a high priority critical load...

Figure 17. Probability of meeting $10 \%$ of highest priority critical load for a large base as a function of the number of stand-alone EDG per building as compared to a microgrid

\section{List of Tables}

Table ES- 1. Annual Hourly Peak Critical Load, Number of Critical Buildings for the Small, Medium, Large, and Very Large Bases Sizes Modeled vi

Table ES- 2. 90\% Confidence Ranges for the Load a Microgrid Must Shed and the Number of Buildings With Critical Load That Will Not Have Power if One Uses a Single Stand-Alone vii

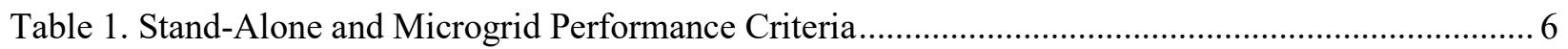

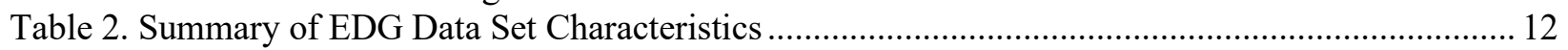

Table 3. MTTF Data Including Mean and 90\% Confidence Intervals for the Three Data Sets................. 13

Table 4. Mean FTS Probabilities and the 90\% Confidence Intervals .................................................. 13

Table 5. Availability Estimates for Different Levels of Maintenance ….................................................... 15 
Table 6. Annual Hourly Peak Critical Load, Number of Critical Buildings, and N Generators for the Microgrid Scenario for the Small, Medium, Large, and Very Large Bases Sizes Modeled

Table 7. 90\% Confidence Ranges for the Probability of Meeting 100\% of the Critical Load for a Microgrid and a Single Stand-Alone Building-Tied EDG at 1, 3, 7, and 14 days 28

Table 8. 90\% Confidence Ranges for the Load a Microgrid Must Shed and the Number of Buildings With Critical Load That Will Not Have Power if One Uses a Single Stand-A 30

Table 9. 90\% Confidence Ranges for the Probability That the 10\% and 25\% Highest Priority Buildings With Critical Loads Will Have Power When Using a Stand-Alone System of Two EDGs Per Building at 7 and 14 D 


\section{Introduction}

\subsection{Background}

The U.S. Department of Defense's (DoD's) fixed installations — or military bases — are a critical element of national security (1). Military bases have long supported the maintenance and deployment of weapons systems and the training and mobilization of combat forces. Increasingly, they perform direct support to combat operations. They also play a critical role in homeland defense and during domestic emergencies can provide support to civil authorities. Today they cannot be assumed to be free from threats; as the recent National Defense Strategy (2) noted, "the homeland is no longer a sanctuary."

Fixed installations cannot operate without energy and they rely on electricity to support critical missions and functions. DoD's fixed installations consumed more than 200,000 billion ${ }^{1}$ BTUs in 2018 (3). The military's use of facility energy carries a hefty price tag: DoD's utility bill is almost $\$ 4$ billion per year. But more important for the military's mission is its reliance on energy entails operational risk as well as cost.

Installations are dependent on a commercial grid that is vulnerable to disruption due to severe weather, physical attacks, and cyberattacks. Power outages are a fact of life. Outages can range in duration from minutes to weeks, and their impact can be geographically limited (a failure in a single feeder line) or widespread (a failure in the bulk transmission system that affects hundreds of thousands of people in multiple states). The risks of blackouts and loss of electric power are not new. Outages of just a few hours are well known, but longer duration outages are becoming more frequent (4). In the United States, these outages are driven by an increasing frequency and intensity of severe storms (thunderstorms, blizzards, hurricanes, and other high-wind events), fires, and increased load demand and strain due to extreme temperature events, including heat waves and polar vortices. These outage threats are increasing due to climate change and unlikely to return to historical norms in the future.

In addition to natural hazards, the commercial grid is vulnerable to manmade threats, both physical and cyber. The fastest growing threat to the electric grid is cyberattacks, in which hackers try to manipulate industrial control and Supervisory Control and Data Acquisition (SCADA) systems to disrupt the flow of electricity. Energy infrastructure has become a major target of cyberattacks (5). More frequent and sophisticated attacks are likely from both nationstates and cyber criminals.

The current default solution for energy assurance and resiliency at military installations relies on emergency diesel generators (EDGs). This is most often accomplished by either a single standalone generator or two generators tied to an individual building. Less common, but with increasing frequency, diesel generators are networked and serve as the primary distributed energy resource (DER) for a microgrid. Today there is no actionable information on the reliability of EDGs and their impact on energy assurance and resiliency. In the absence of such information, military installations cannot quantitively assess their current energy assurance vulnerabilities nor evaluate alternative approaches. Furthermore, as more advanced solutions

${ }^{1}$ This includes both electricity and natural gas. 
involving renewable energy generation and storage evolve, reliability information is required for the current baseline approaches to assess the value of these new solutions.

\subsection{Scope of Study}

The National Renewable Energy Laboratory (NREL) was tasked by DoD's Environmental Security Technology Certification Program ${ }^{2}$ (ESTCP) to develop the information and methodology required to quantify the reliability of EDGs and their impact on the effectiveness of backup power systems being deployed on DoD installations. This report documents the results of that effort.

A comprehensive review of the reliability data literature for both emergency and nonemergency diesel generators was conducted. Based on existing data sets, a new set of reliability probabilities and metrics were developed for the EDGs commonly used on DoD's fixed installations. A technical review of existing methodologies for calculating the probability for a system of diesel generators to meet critical load requirements during outages of various lengths was conducted. Based on the limitations of existing methodologies, a new method was developed that provides predictions for the reliability of systems of EDGs.

A set of scenarios was developed for military installations, and the resulting energy reliability was calculated. The installation energy scenarios include a range of critical load sizes typically found on military installations and realistic hourly load profiles. The scenarios include outages ranging from one hour to two weeks and cover typical conditions found on small national guard and reserve bases up to very large domestic active military installations. These scenarios can serve as screening level benchmarks for the performance expected on fixed installations worldwide. The tool used for this analysis is available, through NREL, for site-specific assessments to evaluate current energy assurance performance and potential future alternative systems.

This study's results have three limitations that should be recognized. All these limitations can be addressed but require site-specific information. First and foremost is the impact of the reliability of the on-base electric distribution system. Outages due to failures in the on-base distribution system will directly impact the performance of a microgrid system. They also impact a base's energy resiliency for standalone generator systems as they can increase the frequency of outages. Reliability of the on-base distribution system can be considered but requires site-specific information and is not generalizable from one installation to another. Second is the direct destruction of generators due to flooding or other physical disturbances. This can be avoided by smart planning and depends on the location of the generators and the local risk of flooding or other physical disturbances. Third is the impact of fuel storage and distribution. Diesel generators require fuel to operate. Lack of availability due to finite storage or limited resupply can curtail a generator-based system. Also, moving fuel from a central storage area to individual generators is limited by manpower and available transportation. This can be a significant constraint at a large installation that uses stand-alone generators. All these site-specific issues can be modeled if the site-specific information is available.

\footnotetext{
${ }^{2}$ Information on the Environmental Security Technology Certification program can be found at https://www.serdpestcp.org/
} 


\section{Installation Energy Assurance}

\subsection{DoD Energy Policy}

DoD's energy policy is "to enhance military capability, improve energy security and resilience, and mitigate costs in its use and management of energy." This policy applies to military bases. It is further articulated in DoD's Installation Energy Management instruction. ${ }^{4}$ The instruction states that DoD components "shall take necessary steps to ensure energy resilience on military installations. DoD Components shall plan and have the capability to ensure available, reliable ${ }^{5}$, and quality power to continuously accomplish DoD missions from military installations and facilities." The instruction further states that the components "shall clearly define, identify, and update critical energy requirements that align to critical mission operations in collaboration with tenants, mission owners, and operators of critical facilities on military installations." Thus, it is a requirement for all military bases to insure they have reliable backup power needed to carry out their critical missions. DoD's policy also explicitly states that "Energy resilience solutions are not limited to traditional standby or emergency generators." Up to now, military installations have lacked the tools and information to quantify "reliable" power. This study addresses that key need for systems comprised of stand-alone generators or microgrid configurations dependent on EDGs.

\subsection{Current Practice}

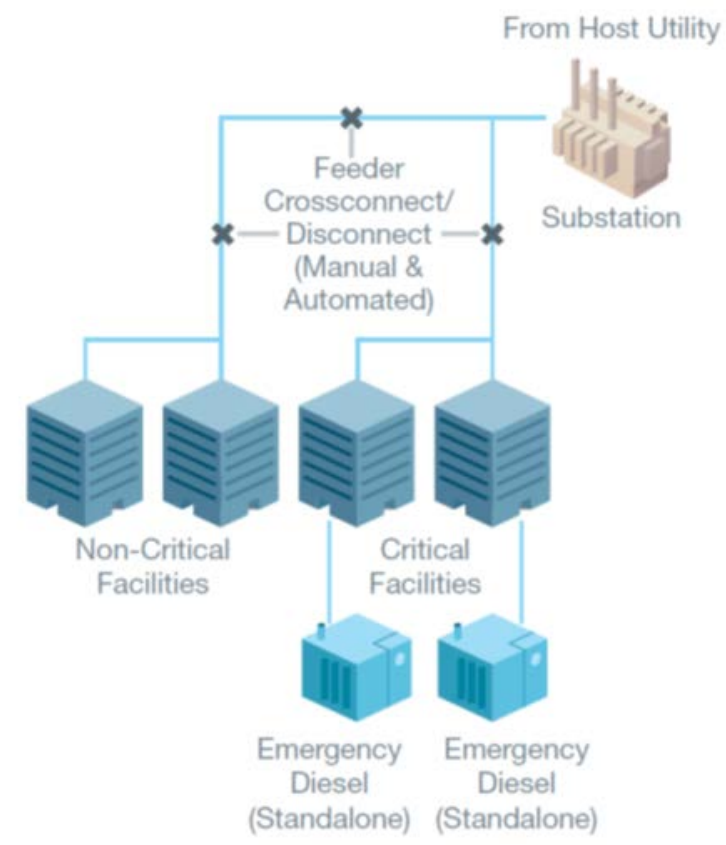

Figure 1. Historical approach—stand-alone generators tied to individual buildings

Stand-Alone Emergency Diesel Generators: DoD has historically relied on stand-alone generators with short-term fuel stockpiles to provide emergency backup power for buildings with critical loads. At every building housing a critical load, a single (stand-alone) backup generator is

\footnotetext{
${ }^{3}$ Energy Policy DODD 4180.01 August 2018.

${ }^{4}$ Installation Energy Management DODI 4170.11 August 2018.

${ }^{5}$ Emphasis added.
} 
hardwired directly to the building. For the highest priority critical loads, two stand-alone backup generators can be deployed to provide a backup to the backup and a higher degree of reliability. Backup generators found on fixed installations are powered by diesel fuel. A base typically has a centrally managed diesel fuel stockpile that contains enough fuel to allow the generators to run for two to seven days. Figure 1 provides a simplified graphical representation of such a system.

Stand-alone generators on a base are diverse and numerous. They can range in size from $10 \mathrm{~kW}$ to $100 \mathrm{~s}$ of $\mathrm{kWs}$. Because the generators are disconnected from one another, each is sized to meet a building's peak load. DoD guidance directs generators to be sized at twice the current engineering estimate for their peak load (oversizing accommodates the uncertainty in the engineering estimate and possible increases in the building's future load). In practice, they are often sized even larger (1).

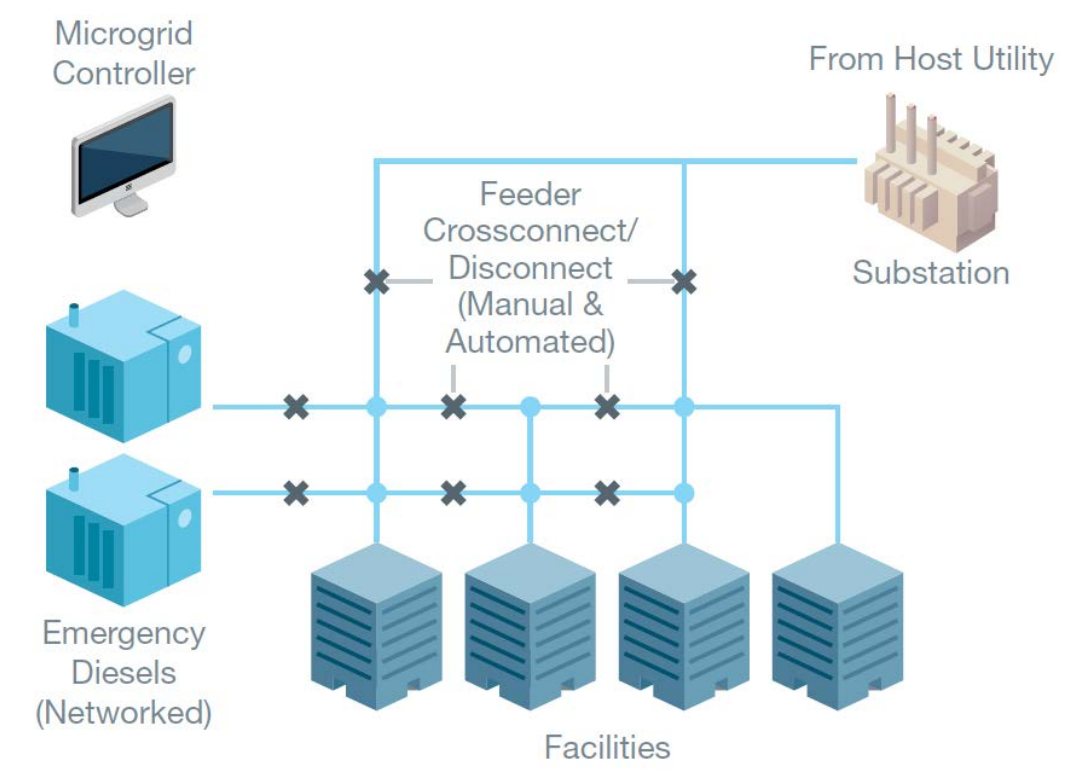

Figure 2. Microgrid with larger networked generators

Microgrid Approach: A microgrid is an alternative way to provide resilient power to a military base. A microgrid is a local system of DERs and electrical loads that can operate as a single entity either in parallel to the commercial (macro) grid or independently (e.g. in "island" mode). Benefits include being used to provide emergency backup power during commercial grid outages or being a source of revenue and savings when connected to the grid. Any on-site power source can serve as a DER, including emergency generators, prime generators, combined heat and power plants, renewables, batteries and other forms of energy. Figure 2 provides a simplified graphical representation of such a system.

Microgrids and stand-alone generators have multiple performance differences (1). There are five key performance criteria that should be considered when assessing the relative value of each system for a specific installation. They are:

1. Reliability - A measure of the likelihood that the critical loads will be supported for a required duration during a grid outage. Stand-alone generators lack $\mathrm{N}+\mathrm{X}$ reliability, 
where $\mathrm{X}$ is the number of independent backups to the first line of defense (stand-alone), which leads to an inherent limited reliability. A microgrid can readily provide a high level of reliability $(\mathrm{N}+1, \mathrm{~N}+2$, or more) because the networked structure ensures that if any single generation asset fails, another one can instantly take its place, and it takes little additional backup power to provide even greater reliability.

2. Flexibility - A system's ability to accommodate changes in the military's electric power needs both during an outage and over longer time periods. Because stand-alone generators are hardwired to the buildings, they can only supply power to that building; the process of moving one to a new location is costly and time-consuming, requiring decommissioning, transport, and recommissioning. Because microgrids are networked, they can respond to changes in electricity needs at little cost as missions change and requirements evolve.

3. Coverage - A system's ability to extend backup power beyond critical loads. Certain intermediate loads both on-base and off-base could advance the mission during an emergency if they had backup power, and some critical loads could get by without a 24/7 level of protection. The reliance on stand-alone generators forces operators to make an "all or nothing" decision: critical loads get 24/7 backup power, and other loads get no backup power. Because a microgrid is at a minimum sized to meet the annual critical peak loads of a base, excess generation is almost always available and can serve any load to which the microgrid is connected, including those loads whose priority falls between "critical" and "noncritical."

4. Dependence on Distribution System - On-base electric distribution systems can fail leading to local outages. Stand-alone generators are not directly impacted by these failures; however, system-level failures lead to an increased need for the stand-alone generator to function and thus do increase the impact of potential failures of the standalone generators. Microgrids are dependent on the on-base distribution system to supply power to critical loads.

5. Vulnerability to Cyberattacks - Stand-alone generators are not required to be networked to any communication system and thus are not vulnerable to a cyberattack. Microgrids depend on an on-base communication system and may be linked to external networks if participating in some off-base electricity markets. Thus, they are susceptible to cyberattack like any other DoD network. 
Table 1 provides a summary of these performance criteria for stand-alone generators and microgrids.

\section{Table 1. Stand-Alone and Microgrid Performance Criteria}

\begin{tabular}{|c|c|c|}
\hline Criteria & Stand-Alone & Microgrid \\
\hline Reliability & - Moderate-to-poor reliability & $\begin{array}{l}\text { - Readily provides a high level of reliability } \\
(\mathrm{N}+1 \text { or more })\end{array}$ \\
\hline Flexibility & $\begin{array}{l}\text { - No ability to meet changing } \\
\text { requirements }\end{array}$ & $\begin{array}{l}\text { - Can respond to changes in mission needs } \\
\text { and priorities }\end{array}$ \\
\hline Coverage & - $\quad$ Covers critical loads only & - Can cover critical and intermediate loads \\
\hline $\begin{array}{l}\text { Distribution } \\
\text { Dependence }\end{array}$ & $\begin{array}{l}\text { Independent of on-base } \\
\text { distribution system }\end{array}$ & $\begin{array}{l}\text { - Vulnerable to failures in on-base distribution } \\
\text { system }\end{array}$ \\
\hline $\begin{array}{l}\text { Cyber } \\
\text { Vulnerability }\end{array}$ & $\begin{array}{l}\text { - Isolated from communication } \\
\text { network }\end{array}$ & - Vulnerable to cyberattack \\
\hline
\end{tabular}




\section{Component Reliability}

In this section we discuss the reliability of a single EDG (6). We first provide a brief introduction to reliability concepts. Then we describe the relevant metrics to quantitatively represent an EDG's reliability. Next, we review the existing empirical data on EDG performance and conclude by providing the mean reliability and uncertainty of an individual EDG's reliability.

\subsection{Reliability Introduction}

"Reliability is the probability that the item will perform its required function under given conditions for a stated time interval" (7). As discussed below, EDGs run very infrequently. Because of this, it is important to precisely define the required function and time intervals we are considering when specifying reliability metrics.

Reliability is the probability that the component will perform its function for time $t$ and is designated $\mathrm{R}(t)$. Equivalently it is the probability that the component will have no failures between the time at which it is required to operate $(t=0)$ and time $t$ in the future. The failure probability is the cumulative distribution function for failures from $t=0$ to time $t$ and is given by:

$$
F(t)=1-R(t)
$$

If we take the first derivative of a cumulative failure distribution function, we obtain the failure probability density function:

$$
f(t)=d F(t) / d t
$$

A common metric used to describe nonrepairable components is the mean time to failure (MTTF).

$$
M T T F=\int_{0}^{\infty} t \times f(t) d t=\int_{0}^{\infty} R(t) d t
$$

Although EDGs are repairable, we use MTTF to define the failure rate as a function of run time. Upon repair, an EDG may again be started, but that constitutes a new run time interval.

Typically, the mean time between failure (MTBF) is used to define the reliability of repairable components. But because EDGs sit idle most of their lifetimes, MTBF cannot be used to estimate the probability of an EDG's runtime failure. We will return to this distinction in the next section.

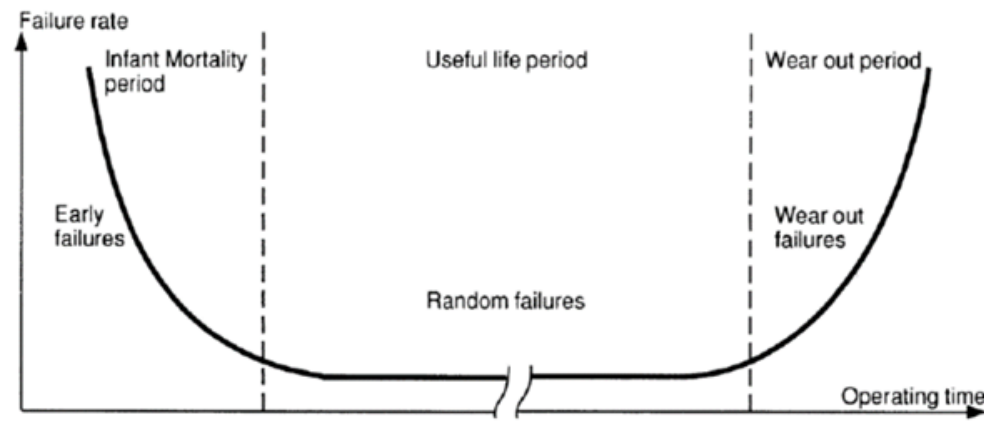

Figure 3. Reliability bathtub model showing a near cnstanr failure rate in the useful life period. 
In this study, we assume that the generator has passed acceptance testing, was properly engineered and manufactured, and is not near the end of its life when it should be replaced. In terms of the reliability literature's "Bathtub Model" (Figure 3), the generator is in its useful life period. During this period, we assume that the failure rate while running, $\lambda$, is constant. If one considers only run time failures, $\mathrm{R}(\mathrm{t})$ is:

$$
R(t)=e^{-\lambda t}
$$

Where:

$$
\lambda=1 / M T T F
$$

\subsection{Emergency Generator Reliability Metrics}

Most energy reliability assessments are concerned with systems or components intended to operate continuously. EDGs run very infrequently and sit in a cold state for most of their lifetimes. The Clean Air Act regulations limit their operations to 200 hours a year for nonemergency use, but most run less than that.

The standard source for reliability data for equipment used in industrial and commercial power systems is the Institute of Electrical and Electronics Engineers (IEEE's) Gold Book (8), recently updated in IEEE's 3006.8 Recommended Practice for Analyzing Reliability Data for Equipment Used in Industrial and Commercial Power Systems (9). IEEE provides summary data on key reliability metrics for hundreds of components. The summary data is based on data from two major collection efforts conducted by the U.S. Army Corps of Engineers Power Reliability Enhancement Program (PREP). As the IEEE standard states, the PREP's "efforts created the most comprehensive facility equipment reliability database in existence." IEEE's earlier reports are identical to the reliability data reported in the U.S. Army standard (10), which also is derived from the PREP database. The Army has not updated their published guidance since 2006 and now relies on the IEEE documentation of its data collection. Both government and commercial assessments of reliability are dependent on the recent IEEE reported results.

For EDGs, the IEEE- and PREP-reported reliability data is inadequate and inappropriate for assessing the performance of EDGs for providing backup power during a grid outage for three reasons:

1. IEEE and PREP only report annual failure rates, which are not relevant for assessing the run time failure rate of an EDG. EDGs only run during a grid outage or for testing, which accounts for a very small fraction of the year. The annual failure rate is sensitive to the number of times an EDG is run (i.e., the number of opportunities it has to fail), which is dependent on the local grid reliability and the testing schedule.

2. EDGs are turned on and off much more frequently than prime generators. EDGs are not kept on hot standby. Thus, the failure to start and carry load is an important characteristic usually not considered in assessing continuous power systems. The probability of an EDG failing to start and carry the load is a well-recognized failure event, but IEEE and the underlying PREP data do not provide this key reliability statistic. 
3. The time to repair reported by IEEE does not include the logistics associated with a repair. It reports only the time required to make the repair once the needed parts and labor are on-site. The time required to obtain parts and have the appropriate technicians on-site is significant and can be larger than the time needed to make the repair.

To properly account for the intermittent use of EDGs, we define an MTTF in terms of the rate of failures while the EDG is running, assuming the EDG has successfully started and carried the load. This can empirically be calculated as:

$$
\text { MTTF }=\frac{\text { total run time }}{\text { number of failures while running }}
$$

This metric is independent of the grid's reliability and the EDG's testing frequency and is dependent only on the EDG cumulative run time. The impact of more frequent grid failures requiring more frequent EDG demands are factored out. We separately consider the probability that the EDG fails to start and carry the load. This can empirically be calculated as:

$$
\text { Failure to Start (FTS) Probability }=\frac{\# \text { failures to start }}{\# \text { of attempts to start }}
$$

These two metrics define the inherent reliability of an EDG (i.e., independent of the grid's reliability and the testing frequency of the unit). Since EDGs are repairable, one often encounters the metric MTBF (8) (9) (10), defined as:

$$
M T B F=\frac{\text { lifetime }}{\text { number of failures }}
$$

which is independent of whether the failure occurred upon start or while running and is dependent on the frequency of demands on the EDG due to testing and grid outages. The MTBF is simply the inverse of the annual failure rate. MTBF or equivalently annual failure rates are provided in the IEEE literature and should not be used when trying to estimate the run-time failure rate of EDGs during a grid outage.

One additional metric we require is the operational availability (OA) of the EDG. This is defined as the probability that the EDG is in service (or available to attempt to provide power) at the start of a grid outage.

$$
O A=\frac{\text { lifetime }- \text { time offline due repairs and maintenance }}{\text { lifetime }}
$$

An EDG could be offline or unavailable due to ongoing repairs initiated due to a failure or due to scheduled maintenance. These out-of-service times are characterized by the mean time to repair (MTTR), which is the mean time associated with unscheduled repairs due to failures, and the mean time to maintain (MTTM), which is the mean time associated with scheduled maintenance activities that require the system to be taken offline. OA is sensitive to maintenance and repair times, as well as the annual failure rates and maintenance schedules. 


\subsection{Data Sources}

To our knowledge, only four data sets are both large enough ${ }^{6}$ and relevant to EDGs to be used to estimate the required reliability metrics discussed previously. While none of these data sets provide information for all four metrics, they can be used together to provide insight on the performance of an EDG during a long-term grid outage. Below, we briefly review these four data sets (11) (12) (13) (14) and summarize their characteristics.

The data collected by the PREP that forms the basis for all reported IEEE reliability results was collected from over 200 sites in the United States and Canada. The sites include military facilities, hospitals, and universities. PREP collects data by surveys from facilities and follows up with site visits when possible. The PREP data for EDGs is divided into two size classes: $<250$ $\mathrm{kW}$ and $250 \mathrm{~kW}-1,500 \mathrm{~kW}$. The PREP data does not include information on the number of attempted starts or run time of the EDGs. Thus, estimates for FTS and MTTF based on run time cannot be constructed. PREP data includes the number of failures as a function of the observation time or, equivalently, the annual failure or MTBF. PREP data also includes detailed data on the time required for maintenance activities and the time to repair in case of failures which can be used to estimate availability. While this data set does not provide metrics for FTS or MTTF, it will be used later in this study to estimate maintenance and repair time to calculate OA.

Maintenance frequency and practices affect an EDG's availability and reliability. PREP rates each site according to the quality of maintenance employed and categorizes the sites into three tiers:

- Above average maintenance is reserved for facilities that followed a scheduled preventative maintenance policy equivalent to the manufacturer's suggested policy; meets National Fire Protection Association (15) or DoD's Unified Facility Criteria (16) recommended maintenance practices; uses specialized equipment tests (thermograph, vibration analysis, oil analysis); and has complete spare parts kits for the equipment. 25\% of the PREP sites employ above average maintenance.

- Average maintenance also rigorously follows recommended maintenance schedules but does not use specialized equipment or have complete spare parts on hand. 57\% of PREP sites employ average maintenance practices.

- Below average maintenance either has no formal maintenance policy and schedule or fails to follow one. $17 \%$ of PREP sites employ below average maintenance.

For the purpose of this study, we partition maintenance practices into two classes: wellmaintained EDGs, which include both average and above average maintenance practices; and poorly maintained EDGs, which are equivalent to below average maintenance. An EDG on a military base that rigorously follows Unified Facility Criteria guidance is well-maintained.

A study conducted in Hong Kong (11) reported data on 147 EDGs monitored for an average of five years. The data was collected via a generator reliability survey followed by site visits when feasible. The EDGs were used in commercial, residential, industrial, and institutional settings to provide backup power during a grid failure. They ranged in size from $80 \mathrm{~kW}$ to $1,500 \mathrm{~kW}$, which is typical of EDGs used on military bases. The distribution of the sizes was not reported. The

\footnotetext{
${ }^{6}$ Given the low probability of failure, a data set must be large enough to yield a result in which the confidence intervals for the key metrics are not meaningless.
} 
authors reported that poor maintenance practices resulted in high reported FTS of $1.65 \%$ and an MTTF of only 61 hours. This data set provides a benchmark for EDGs in the below average or poorly maintained category. Obviously, there is a wide range of maintenance practices that are classified as poor. Thus, this case should be viewed as only one example.

In the United States, the Nuclear Regulatory Commission (NRC) requires that the performance data on EDGs that support nuclear power plants be reported routinely. Like all EDGs, those at nuclear power plants do not operate all the time. They are required to operate when the grid power is down and during shutdown periods. The demands and run hours are reported on a quarterly or semi-annual basis, and existing regulations established the requirements for testing of these on-site power sources. Therefore, an extensive database on these EDGs exists. Recent analysis of this database (13) has calculated the EDGs' reliability metrics. All demand types on the EDGS are considered, including both testing, as well as operational. These EDGs range in size from $50 \mathrm{~kW}$ to $499,999 \mathrm{~kW}$, and most are considerably larger than those used on military bases. The sizes of the EDGs in this database are summarized in Figure 4.

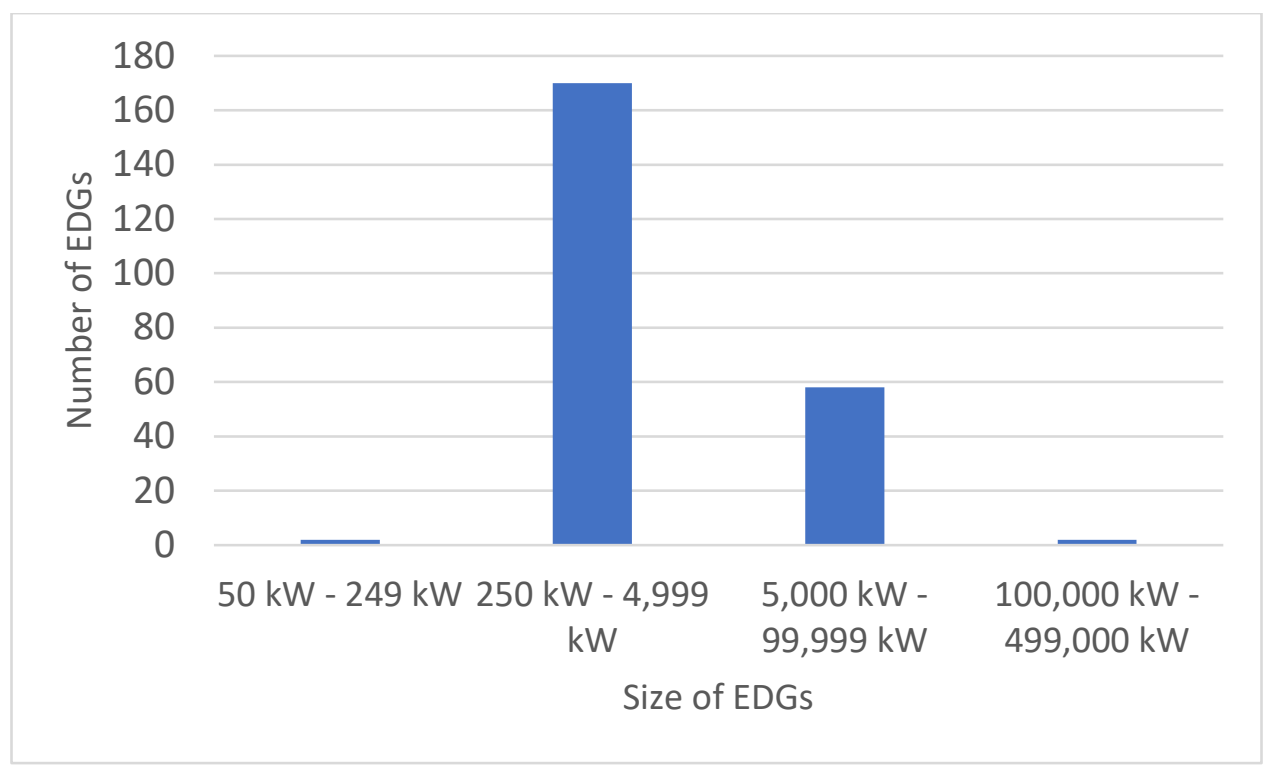

Figure 4. EDG size distribution in NRC database

Since this data set represents all EDGs used at U.S. nuclear power plants, it provides insight into an industry that requires high reliability, and the EDGs are assumed to be well-maintained. They reported an FTS of $0.66 \%$ and a MTTF of 636 hours, considerably better than the results for the smaller poorly maintained EDGs in the Hong Kong study. Due to the EDG size distribution of this data set, direct comparison of their reliability performance for military applications cannot be done. We include them in this study to provide an example of reliability for another industry (i.e., nuclear power).

The final data set we consider was collected in support of a Ph.D. thesis (14) supported by the U.S. Navy. The research was intended to provide facility managers with data to optimize the staffing level and generator maintenance. The scope of the study was limited to modern, high-efficiency, low-emission generator sets. Maintenance logs that followed current government regulations were 
collected and entered into a structured database. The sample population included EDGs between $10 \mathrm{~kW}$ and 2,000 kW. Figure 5 shows the EDG size distribution for this database.

The database contains information on run times, as well as attempted starts and failures. Detailed information on the maintenance practices were recorded but do not include data on downtime due to maintenance time or repair time due to failures. This data set will be used to provide a benchmark for EDGs used on military installations that are well-maintained. Metrics calculated from this dataset are reported and discussed in the following section.

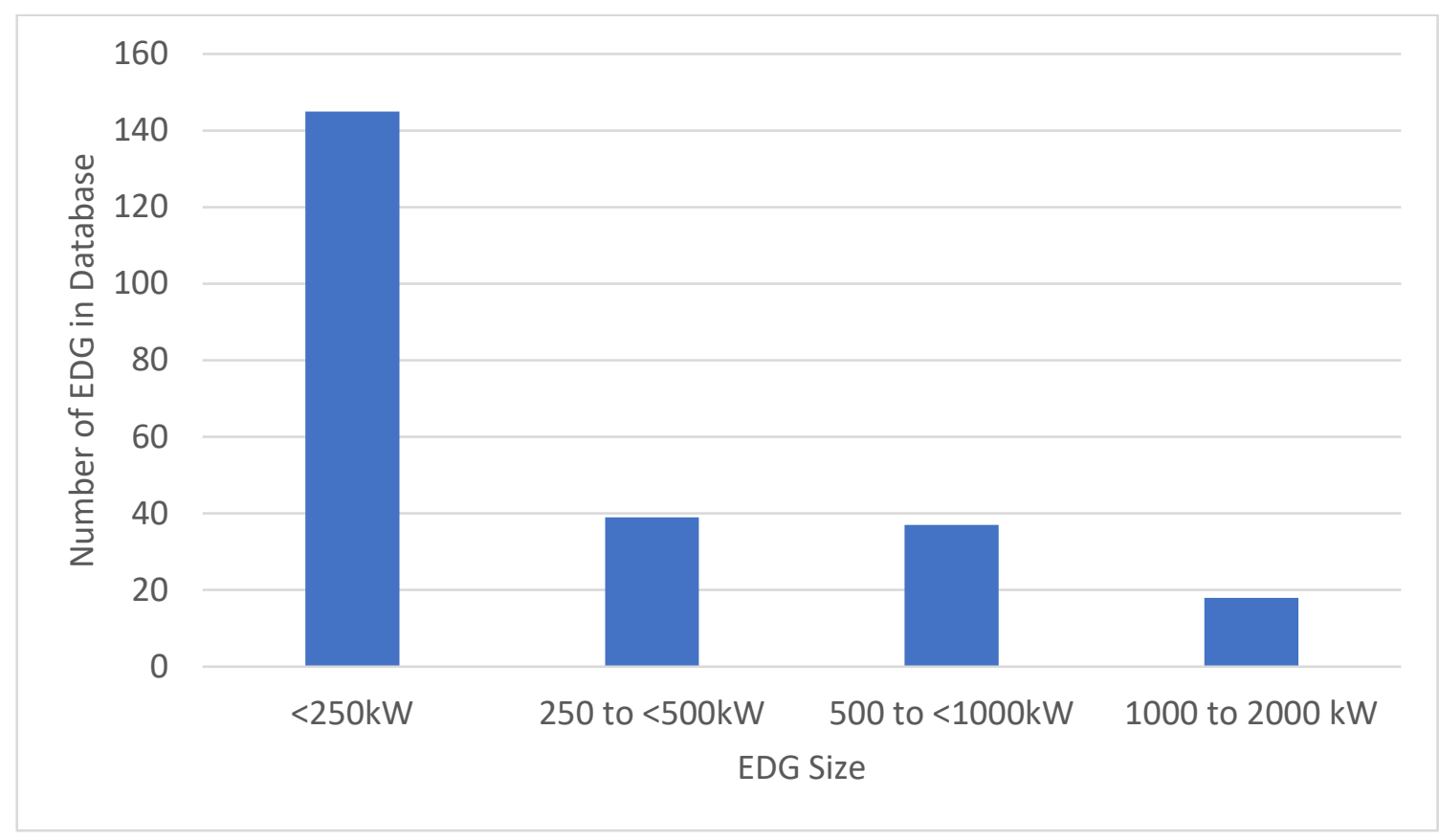

Figure 5. EDG size distribution in the Fehr database

The characteristics for these four data sets are summarized in Table 2.

Table 2. Summary of EDG Data Set Characteristics

\begin{tabular}{|c|c|c|c|c|}
\hline Source & \# EDGs & $\begin{array}{c}\text { EDG Years } \\
\text { of } \\
\text { Observation }\end{array}$ & $\begin{array}{c}\text { Available or Derivable } \\
\text { Metrics }\end{array}$ & Comments \\
\hline PREP & 304 & 2,298 & $\begin{array}{ll}\text { - } & \text { MTBF } \\
\text { - } & \text { MTTR } \\
\text { - } & \text { MTTM } \\
\text { - } & \text { OA }\end{array}$ & $\begin{array}{ll}\text { - } & \text { Representative size EDG } \\
& (\leq 1,500 \mathrm{~kW}) \\
\text { - } & \text { Mixed maintenance }\end{array}$ \\
\hline Hong Kong & 147 & 790 & $\begin{array}{ll}- & \text { MTTF } \\
- & \text { MTBF } \\
- & \text { FTS }\end{array}$ & $\begin{array}{ll}\text { - } & \text { Representative size } \\
& \text { EDGS }(80 \mathrm{~kW}-1,500 \mathrm{~kW}) \\
\text { - } & \text { Poorly maintained }\end{array}$ \\
\hline NRC & 232 & 1,790 & $\begin{array}{ll}\text { - } & \text { MTTF } \\
\text { - } & \text { MTBF } \\
\text { - } & \text { FTS }\end{array}$ & $\begin{array}{ll}\text { - } & \text { Large EDGS (most }>1 \\
& \text { MW) } \\
\text { - } & \text { Well-maintained }\end{array}$ \\
\hline Fehr & 239 & 1,281 & $\begin{array}{ll}- & \text { MTTF } \\
- & \text { MTBF } \\
- & \text { FTS } \\
\end{array}$ & $\begin{array}{ll}- & \text { Representative size } \\
& \text { EDGS }(10 \mathrm{~kW}-2,000 \mathrm{~kW}) \\
\text { - } & \text { Well-maintained }\end{array}$ \\
\hline
\end{tabular}




\subsection{Emergency Generator Reliability}

The MTTF is the most important EDG reliability parameter when looking at EDG performance periods from days to weeks. Over long duration outages, failures while running dominate the overall reliability.

We have analyzed the recently collected Fehr data set to determine the MTTF for wellmaintained EDGs. As shown in (14), this data contains no statistically significant evidence that the generator's make, model, or size $(10 \mathrm{~kW}-2,000 \mathrm{~kW})$ has any significant impact on reliability. Using a simple frequentist analysis, ${ }^{7}$ the MTTF and its $90 \%$ confidence intervals are provided in Table 3. This information is compared to results from the NRC and Hong Kong data sets described above that provide information on failures as a function of run time (the PREP data does not contain information on run times).

Table 3. MTTF Data Including Mean and $\mathbf{9 0 \%}$ Confidence Intervals for the Three Data Sets

\begin{tabular}{|l|l|l|l|}
\hline Data Source & MTTF Low Value & MTTF Mean Value & MTTF High Value \\
\hline Fehr & 1,180 hours & 1,662 hours & 2,410 hours \\
\hline NRC $(13)$ & 568 hours & 636 hours & 714 hours \\
\hline Hong Kong & 53 hours & 61 hours & 71 hours \\
\hline
\end{tabular}

The 90\% confidence intervals do not overlap. The Fehr and Hong Kong data sets involve similar size and types of EDGs. The Fehr and Hong Kong data are for EDGs with significantly different levels of maintenance. The well-maintained EDGs in the Fehr data set have MTTFs over 20 times longer than seen in the poorly maintained Hong Kong data set. The NRC data set includes much larger EDGs. Whether their relative MTTF (between the other two data sets) is due to the size of the generators or the maintenance practices in the nuclear industry cannot be determined.

As stated above, EDGs are not kept on hot standby and must start and transfer power to the load when called upon during a grid outage. FTS is a rare phenomenon but significant enough to warrant its inclusion in reliability assessments. The NRC and Hong Kong data sets report number of attempted starts and failures to start. The NRC divides its failures to start into two classes: immediate failures and failures to start and carry load. ${ }^{8}$ We include both events. For the Fehr data set, 44 FTS were observed for the 239 EDGs monitored. But the number of attempted starts was recorded only for 35 of the 239 EDGs in the data set. Three of these EDGs were installed for less than two months and were still undergoing initial testing. The average number of starts per year was 26.7, consistent with common practices. Applying this simple but crude estimate for the remaining EDGs yields 34,134 attempted starts over the observation period for the 239 EDGs, resulting in a mean FTS of $0.13 \%$.

Table 4. Mean FTS Probabilities and the $90 \%$ Confidence Intervals

\begin{tabular}{|l|l|l|l|}
\hline Data Source & Low Value FTS & Mean Value FTS & High Value FTS \\
\hline Fehr & $0.10 \%$ & $0.13 \%$ & $0.17 \%$ \\
\hline NRC & $0.26 \%$ & $0.66 \%$ & $1.20 \%$ \\
\hline Hong Kong & $1.44 \%$ & $1.65 \%$ & $1.88 \%$ \\
\hline
\end{tabular}

\footnotetext{
${ }^{7}$ NRC (U.S. Nuclear Regulatory Commission). “About the Reliability Calculator.” Last modified March 6, 2019 https://nrcoe.inl.gov/radscalc/.

${ }^{8}$ Failure to carry load includes any failure that occurs within one hour of starting.

9 These are the FTS values that include both the immediate FTS and the failures to carry load.
} 
Statistics on the FTS with $90 \%$ confidence intervals are shown in Table 4 for all three data sets (the PREP data contains no information on attempted starts). Like the MTTF metrics, wellmaintained EDGs are much more reliable with the FTS probability an order of magnitude lower than for poorly maintained EDGs. The larger EDGs used in the nuclear industry have FTS and carry load roughly midway between the other two data sets. Again, the cause for this difference may be due to the size of the EDGs or maintenance practices.

The operation availability of an EDG is dependent on the annual failure rates, the time it takes to repair a failure, and the time the EDG is out of service due to scheduled maintenance activities. The PREP database contains information on the repair and maintenance times. The published mean time to repair does not include the logistics time and is not relevant for calculating an OA; however, the underlying database does include the needed information. Figure 6 shows the distribution of repair times, including logistics for all EDGs in the PREP database. Due to PREP reporting from earlier data collection efforts, PREP characterized a subset of observations by the subset's mean, which falls in the 16-24-hour interval. Thus, the data artificially appears as a bimodal distribution.

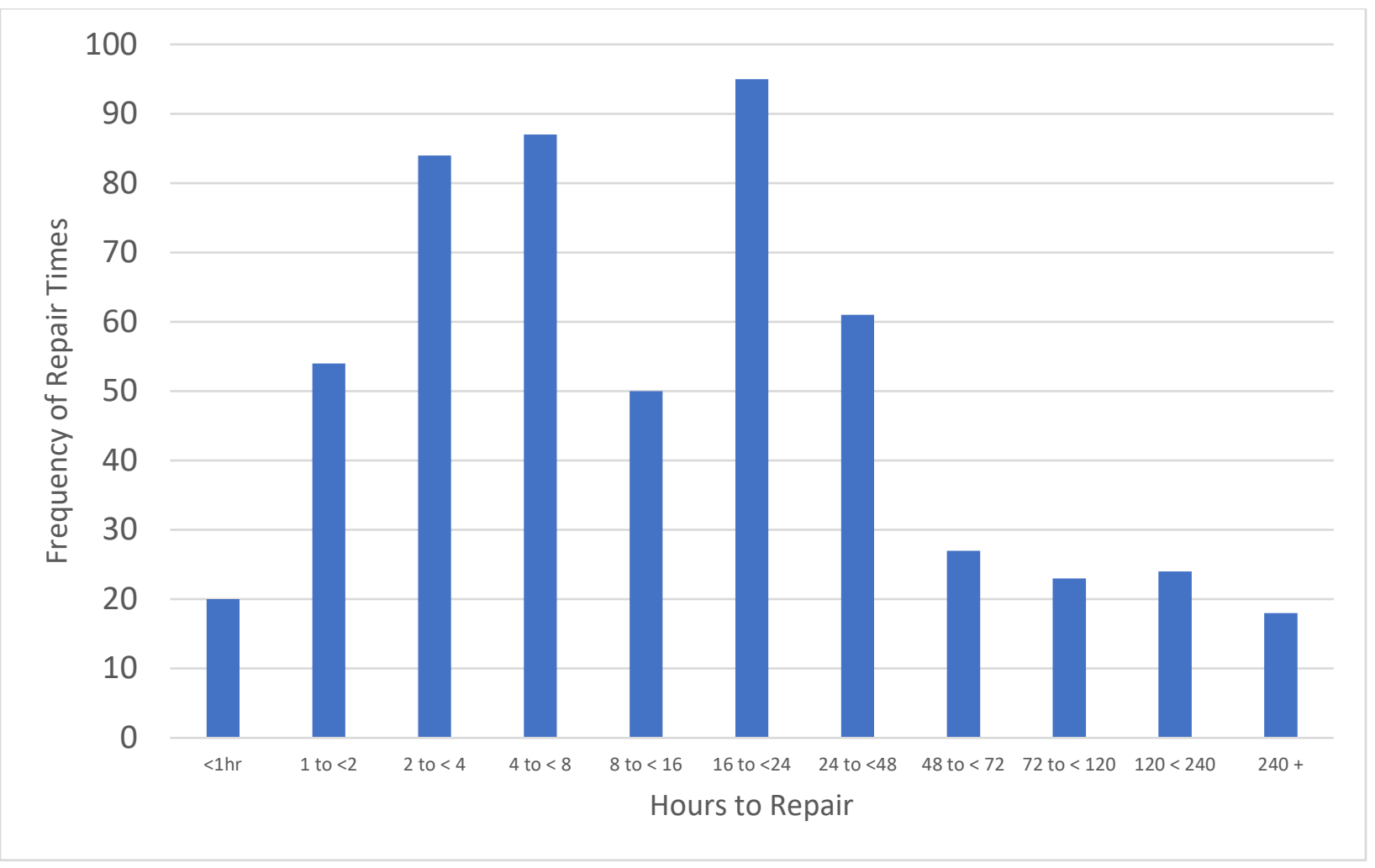

Figure 6. Distribution of repair times, including logistics for EDGs after a failure

The MTTR is 37 hours if we eliminate a single outlier, which was nearly 2,000 hours and more than twice the next-longest repair time. The MTTM is narrowly distributed, with a mean value of 1.7 hours ${ }^{10}$. Most outages are due to extreme weather events, and scheduled maintenance is often delayed when severe weather is expected. For assessing the performance of an EDG to provide

\footnotetext{
${ }^{10}$ PREP database
} 
power during extended outages, we will ignore this short duration of an EDG being unavailable due to scheduled maintenance. Providing power for very short outages (typically less than 15 minutes) is accomplished by an uninterruptable power supply rather than an EDG, which is not the subject of this study.

Under these assumptions, the OA of an EDG or the probability an EDG can attempt to provide backup power can be calculated from annual failure rates and repair times (ignoring scheduling maintenance downtime) from:

$$
O A=\frac{\text { lifetime }- \text { time offline due repairs }}{\text { lifetime }}
$$

If we divide both the numerator and denominator by the number of failures, we find:

$$
O A=\frac{M T B F-M T T R}{M T B F}
$$

Table 5 shows estimates for availability for modest-sized EDGs $(<2,000 \mathrm{~kW})$ that are well or poorly maintained. The MTTR is taken from the PREP data and applied to the Fehr and Hong Kong data sets. The differences in OA are due to the failure rates or, equivalently, the number of required repairs.

\section{Table 5. Availability Estimates for Different Levels of Maintenance}

\begin{tabular}{|l|l|}
\hline Maintenance & OA \\
\hline Well-Maintained & $99.98 \%$ \\
\hline Poorly Maintained & $99.84 \%$ \\
\hline
\end{tabular}

These high availabilities reflect the small number of runs per year of an EDG, and, thus, the small number of potential failures per year.

Combining these reliability metrics, the reliability of a single EDG at time $t$ is given by:

$$
R(t)=O A \times(1-F T S) \times e^{-t / M T T F}
$$

We use the estimates listed previously from the Fehr and Hong Kong data sets to model modestly sized EDGs $(<2,000 \mathrm{~kW})$ that are well-maintained or poorly maintained and compare these to the results for larger EDGs used in the nuclear industry. Figure 7 shows the expected reliability for a single EDG for outages that range from one hour to two weeks (336 hours). 


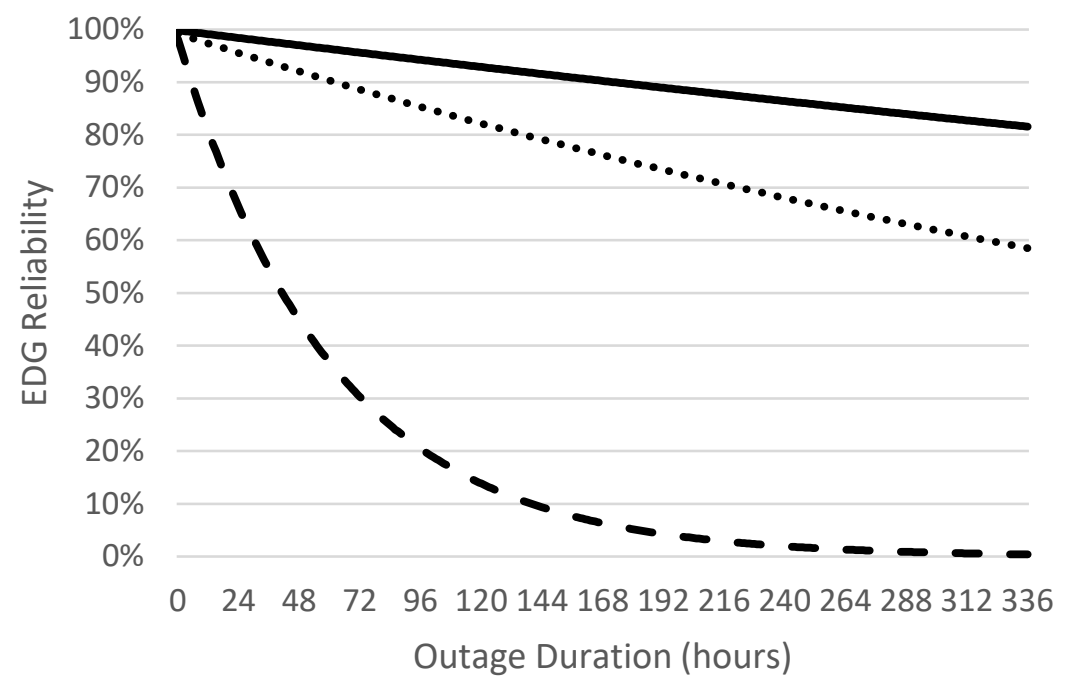

Well-Maintained $\ldots . .$. NRC — - Poorly Maintained

Figure 7. The reliability of a single EDG over two weeks (336 hours)

In Figure 8, the same reliability results for outages up to 12 hours are shown to clearly illustrate the impact of different probabilities for FTS.

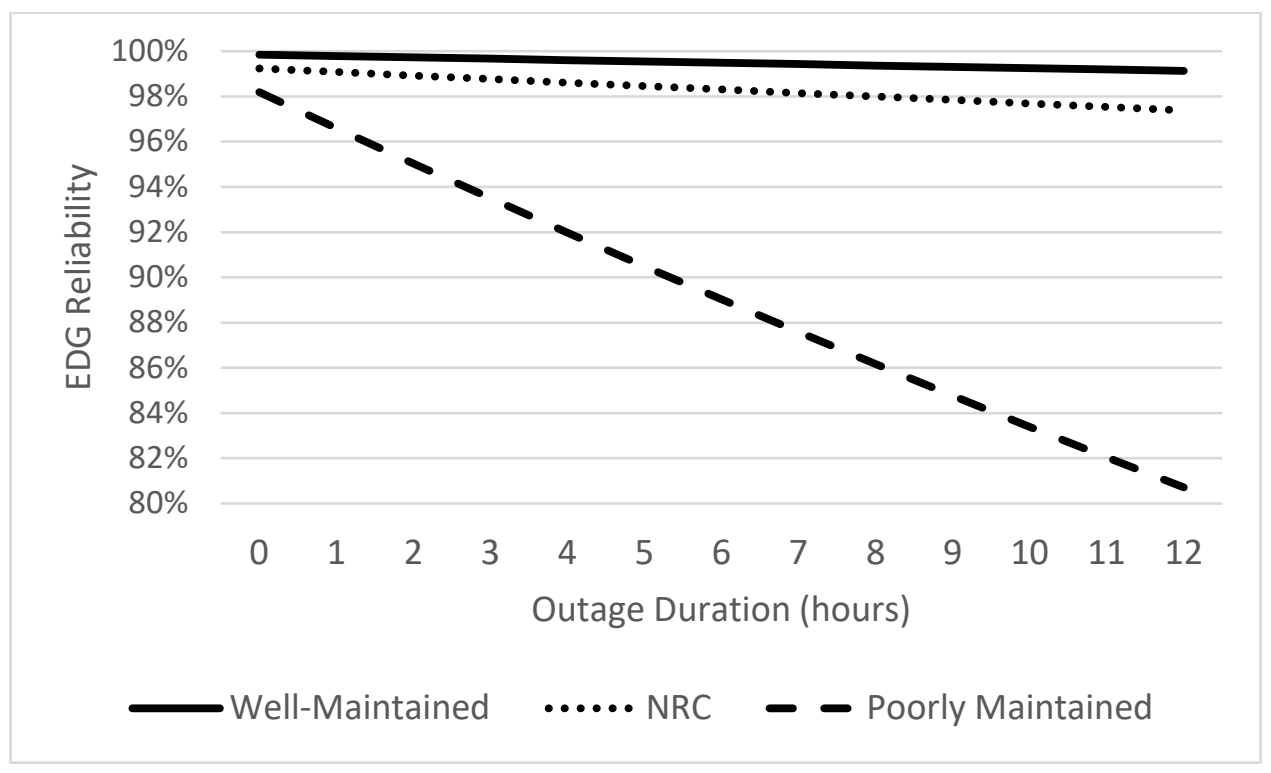

Figure 8. The reliability of a single EDG for outages less than half a day (12 hours) 
Figure 9 shows the reliability range expected for a well-maintained EDG with its $90 \%$ confidence intervals.

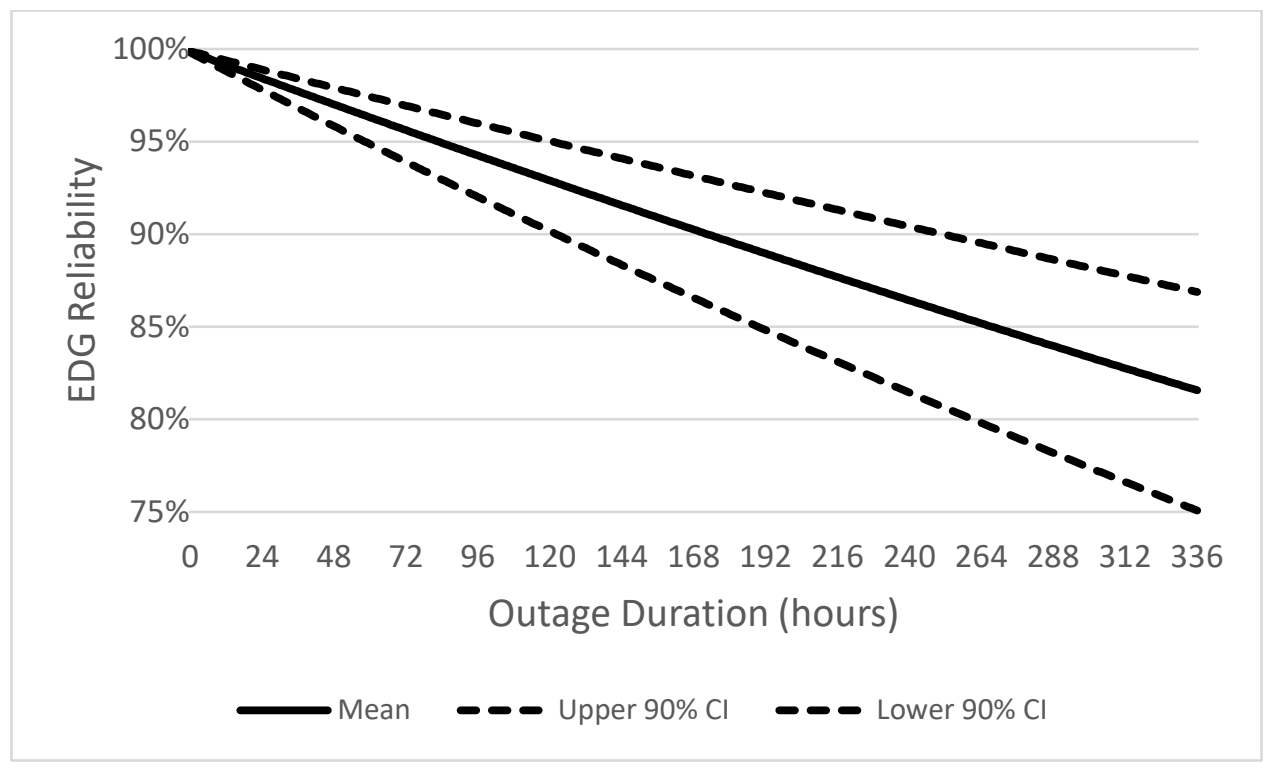

Figure 9. Mean and $\mathbf{9 0 \%}$ confidence interval reliabilities for a well-maintained EDG for outages up to two weeks (336 hours)

Not surprisingly, a poorly maintained EDG is unlikely to provide power for durations longer than a few days, and it has reliabilities of only $80 \%$ at 12 hours. These figures reinforce the importance of following the current guidance on EDG maintenance. But even well-maintained EDGs have a reliability of 92\%-96\% for providing backup power for four days (96 hours), $90 \%$ $95 \%$ at one week, and $75 \%-87 \%$ at two weeks (required for critical loads at some military facilities). Thus, a single EDGs reliability limits their ability to provide a robust source of emergency power for critical loads over multiday outages. 


\section{System Reliability}

Building-tied EDGs and microgrids require different approaches for assessing reliability. Buildingtied EDGs can be assessed using a simple fault tree approach, while networked EDGs require a more sophisticated analysis. Although the primary purpose of a microgrid is to provide power to the critical loads during a grid outage (17) (18), almost all the existing analysis and modeling tools to design and assess microgrid performance do not calculate a microgrid's reliability due to the nonperfect reliability of the DERs that power the system (19). The rare examples of microgrid reliability assessment in the literature are conceptual (20) or use complex Monte Carlo simulations (21). None of them factor in the realistic reliabilities of EDGs that drive the reliability performance of currently deployed microgrids. Below we first describe a simple fault tree analysis sufficient for estimating the reliability of a system composed of stand-alone EDGs and next describe an approach that allows one to estimate the reliability of a microgrid based on EDGs. In both cases, we assume that if an EDG fails at the start or during and outage it is not repaired during the outage. The MTTR of an EDG is 37 hours for all failures (see Figure 6). In the case of a long duration grid outage, we expect the ability to respond to an EDG failure will be worse. Thus, it is unlikely during these extreme events parts and staff will be readily available to diagnose the failure and repair the EDG.

\subsection{Building-Tied}

We consider building-tied systems with one and two generators per building. In the first case, the building loses power when the generator fails while in the second case the building loses power only when both generators fail. Military installations often used a combination of these configurations. Most buildings have one EDG providing backup power and subset of buildings with the highest priority critical load will have two EDGs. We will return to this issue and illustrate its impact in Section 5, but in this section treat them as two separate systems to show the range of building-tied system performance. Providing two EDGs for every building requiring power during a grid outage would be prohibitively expensive.

A generator can fail due to being unavailable at the start of an outage, being available but failing to start, and starting but subsequently failing to run. The reliability that a single generator tied to a building survives an outage of duration $d, R_{1}(d)$, is given by the reliability for the generator as a component:

$$
R_{1}(d)=O A \times(1-F T S) \times e^{-d / M T T F}
$$

If two generators are connected to a building, then the system survives if at least one of the generators survives. The survival probability is then calculated by determining the likelihood that the first or second generator survives, which is given by:

$$
R_{2}(d)=2 * R_{1}(d)-R_{1}(d) * R_{1}(d)
$$

The situation is slightly more complicated if one considers a military installation where critical loads occur in multiple buildings. If we consider b buildings with critical loads, the probability every building has power during an outage, $P A B_{i}(d)$, is:

$$
P A B_{i}(d)=R_{i}(d)^{b}
$$

because each building is independent. Where the subscript $i$ indicates if it is one or two EDGs tied to each building. 
An alternative way to view these results is to calculate the number of buildings that have power during an outage. Assume we have $b$ buildings with critical loads on the base each with a survivability $\mathrm{R}_{i}(d)$. The probability, $\mathrm{P}(k, b)$, that $k$ buildings, have power during an outage at time $d$ is given by the binomial distribution:

$$
\mathrm{P}_{i}(b, k, d)=\left(\begin{array}{l}
b \\
k
\end{array}\right) R_{i}^{k}\left(1-R_{i}\right)^{b-k}
$$

The mean fraction of buildings with power is equal to the mean value of the binomial distribution divided by the total number of buildings and the mean fraction of buildings without power or the expected power lost in terms of fraction of buildings is:

$$
\operatorname{EPLB}_{i}(d)=1-R_{i}(d)
$$

which is independent of the number of buildings.

\subsection{Microgrid}

Calculating the reliability of a microgrid system is more involved than calculating the reliability of a single building-tied EDG. Building-tied generators are sized to meet the peak building load, and the system reliability does not depend on the underlying load profile. The EDGs that power a microgrid can supply power to any building on the microgrid network. Thus, the amount of redundancy at each hour depends on the critical load at that hour. Critical loads vary with season, day of week, and time of day. Consider a large military base with an annual peak hourly critical load of $10 \mathrm{MW}$. A microgrid system powered by a set of $750 \mathrm{~kW}$ centralized EDGs would require at least 14 EDGs to meet that peak load. Typically, a microgrid would be designed to have an $\mathrm{N}+1$ configuration (15 EDGs) to meet the peak critical load and provide some redundancy. Figure 10 displays the critical load profile for this military base in terms of kWhr and units of EDG capacity.

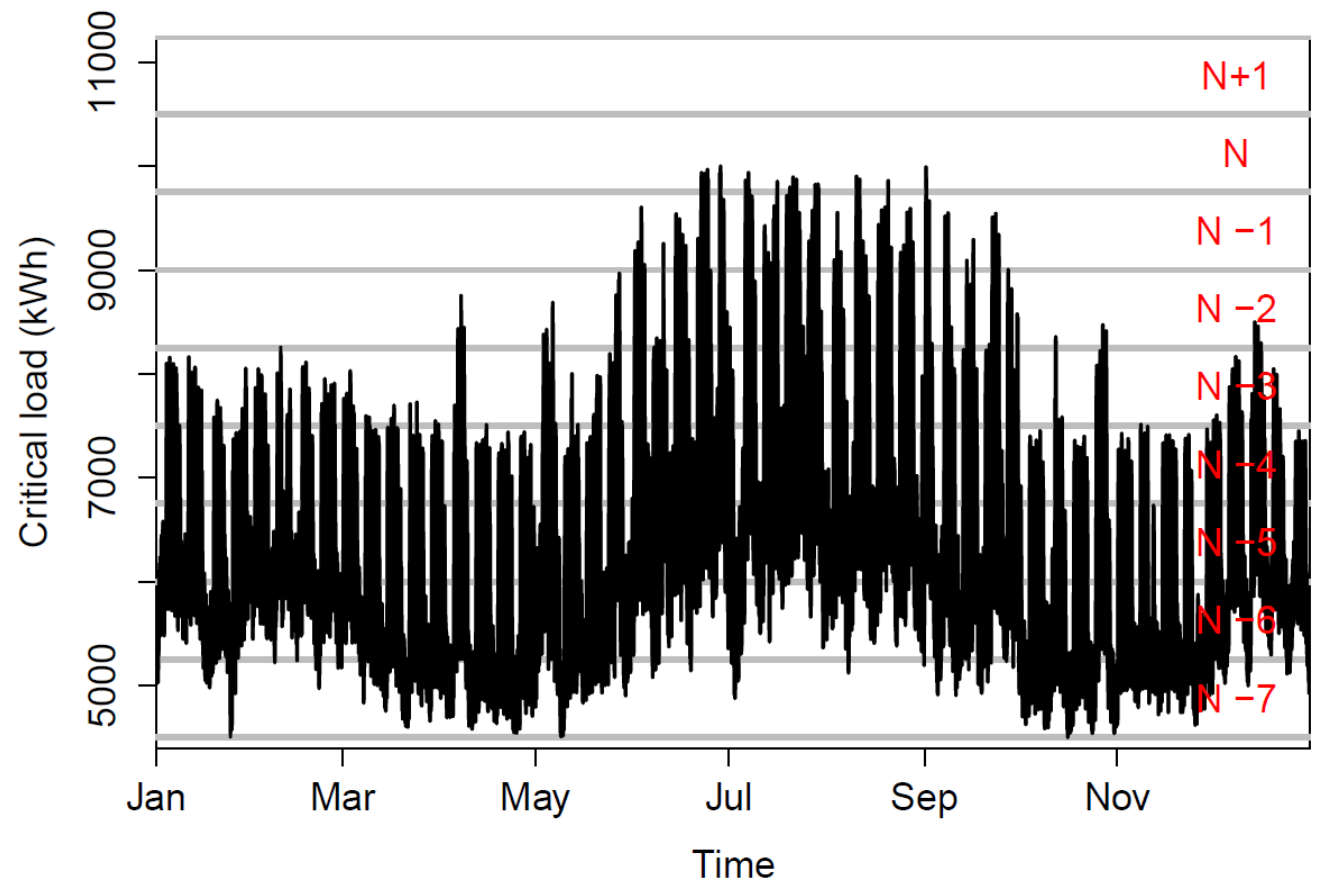

Figure 10. Critical load profile for large base in units of kWh and EDG redundancy 
During most times of the year, multiple EDGs would need to fail for the microgrid system to be unable to meet the load requirement.

Figure 11 shows the distribution of load for this large base over a year ordered by size. The microgrid system for this large base has $\mathrm{N}+1$ reliability for the peak load but has $\mathrm{N}+5$ or more redundancy for more than $80 \%$ of the year. This illustrates why microgrids are an inherently more reliable system than building-tied systems.

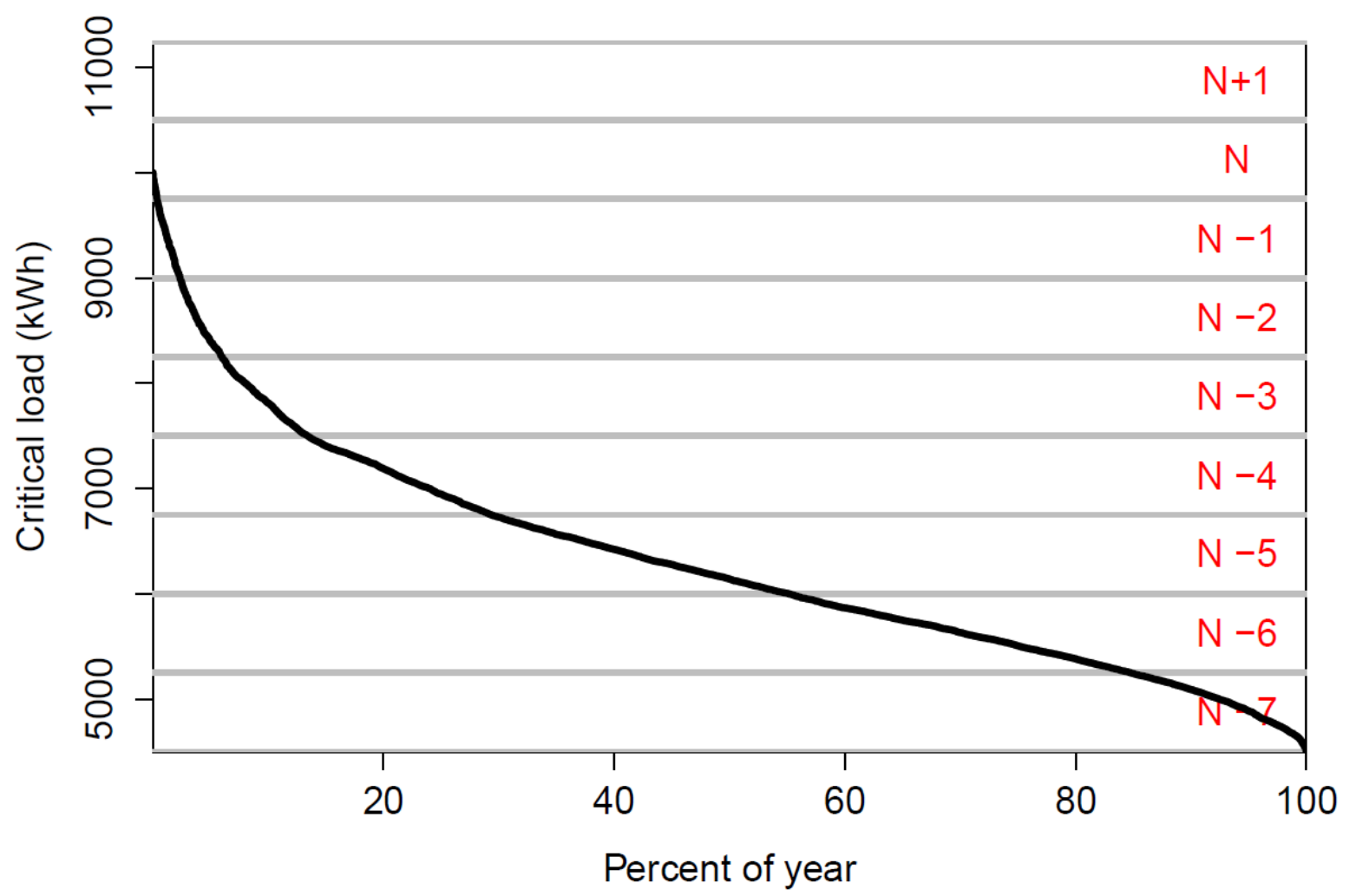

Figure 11. Critical load for large base ordered by load size

To determine the likelihood of survival and the expected critical load shed, we take into account the variability of critical load across the year. Because we model the likelihood of survival hour to hour, we use a discrete representation of component reliability for the microgrid analysis in place of the continuous formulation used for individual buildings. Given a microgrid with $N$ generators, the probability that $n$ generators will be available, and start in hour zero of the outage is given by $P(0, n)$ :

$$
P(0, n)=\left(\begin{array}{l}
N \\
n
\end{array}\right)(O A *(1-F T S))^{n} *(1-O A *(1-F T S))^{N-n}
$$

Which is simply the combinatorics formula for $n$ generators being available and not failing to start, and $N-n$ generators being unavailable or failing to start. We assume all EDGs try to start at the beginning of the grid outage. Although all might not be required, EDGs are ideally run at less than $100 \%$ capacity. Typical guidance recommends the optimal load for a generator should be in the range of $50 \%$ to $80 \%$. The average hourly critical load for our cases is on the order of 50 to $60 \%$ and peak load is between $80 \%$ and $90 \%$ if all EDG are running. Starting all EDGs at 
the beginning of a grid outage and allowing them to continue to run ensures the EDGs are properly loaded. Given $n$ generators are currently running, the probability that $n^{\prime}$ generators are running in the next hour is given by $p\left(n, n^{\prime}\right)$ :

$$
p\left(n, n^{\prime}\right)=\left(\begin{array}{l}
n \\
n^{\prime}
\end{array}\right)(1-F T R)^{n^{\prime}} * F T R^{n-n^{\prime}}
$$

where FTR is the discrete hourly version of exponential decay rate. The probability of $n$ generators still operating after $d$ outage hours, denoted $P(n, d)$, can be found using a Markov matrix, as follows:

$$
\left[\begin{array}{c}
P(0, d) \\
P(1, d) \\
\vdots \\
P(N, d)
\end{array}\right]=\left[\begin{array}{cccc}
1 & p(n, 0) & \cdots & p(N, 0) \\
0 & p\left(n, n^{\prime}\right) & \cdots & p\left(N, n^{\prime}\right) \\
\vdots & \vdots & \ddots & \vdots \\
0 & \cdots & 0 & p(N, N)
\end{array}\right]^{d}\left[\begin{array}{c}
P(0,0) \\
P(1,0) \\
\vdots \\
P(N, 0)
\end{array}\right]
$$

The amount of curtailed load in a given hour depends on both the critical load and the total capacity of generators operating in that hour. The curtailed load for an outage starting at time $t$, in outage hour $d$, given $n$ currently working generators, each with a capacity of $k$, is given by:

$$
C(n, d, t)=\max (0, L(t+d)-k * n)
$$

Where $L(t+d)$ denotes the critical load in hour $t+d$ and $C$ denotes the amount of load curtailed. Assuming outages occur throughout the year with equal frequency, then the microgrid's expected percent of load shed for an outage of duration $d$ is:

$$
E P L S_{m}(d)=\left(\frac{1}{L(t+d) * 8760}\right) \sum_{t=1}^{8760} \sum_{n=0}^{N} P(n, d) * C(n, d, t)
$$

The above equation says that the expected load shed in outage hour $d$ is the sum of curtailed load across the possible number of working generators weighted by the probability that that number of generators is working in outage hour $d$. It is the microgrid equivalent for fraction of buildings without power, $E P L B_{i}(d)=1-R_{i}(d)$, in the case of building-tied systems.

To calculate the probability that all buildings have power, we need to determine the likelihood that no load is curtailed for the entirety of the outage. The procedure for this calculation is very similar to the one described above, but we remove outages in each hour that do not have sufficient capacity to meet load. The probability of survival, which for the microgrid is denoted $S_{m}(n, d, t)$, is determined iteratively by the following two steps:

$$
\begin{aligned}
{\left[\begin{array}{c}
S^{\prime}(0, d, t) \\
S^{\prime}(1, d, t) \\
\vdots \\
S^{\prime}(N, d, t)
\end{array}\right] } & =\left[\begin{array}{cccc}
1 & p(n, 0) & \cdots & p(N, 0) \\
0 & p\left(n, n^{\prime}\right) & \cdots & p\left(N, n^{\prime}\right) \\
\vdots & \vdots & \ddots & \vdots \\
0 & \cdots & 0 & p(N, N)
\end{array}\right]\left[\begin{array}{c}
S_{m}(0, d-1, t) \\
S_{m}(1, d-1, t) \\
\vdots \\
S_{m}(N, d-1, t)
\end{array}\right] \\
S_{m}(n, d, t) & =\left\{\begin{array}{ccc}
0 & C(n, d, t)>0 \\
S^{\prime}(n, d, t) & C(n, d, t)=0
\end{array}\right.
\end{aligned}
$$


Where the initial survival conditions $S_{m}(n, d, t)=P(n, 0)$. In other words, for each hour we determine the likelihoods of having $n$ working generators and then set the probability of survival to zero for systems that have insufficient generation capacity to meet critical load. The probability that all the critical loads are supported by the microgrid, $P A L_{m}(d)$, for an outage of duration $d$ is then calculated as:

$$
P A L_{m}(d)=\left(\frac{1}{8760}\right) \sum_{t=1}^{8760} \sum_{n=0}^{N} S_{m}(m, d, t)
$$

This is the microgrid equivalent for load of the building-tied system probability for buildings given by $P A B_{i}(d)=R_{i}(d)^{b}$. 


\section{Model Installations}

DoD manages real property in all 50 states, 8 U.S. territories, and 45 foreign countries. ${ }^{11}$ This includes over 270,000 buildings on hundreds of installations worldwide. Military installations vary in size and energy demand. They range from small bases that have only a few hundred thousand square feet of building space to extremely large installations with over 20 million square feet. There is no one case that represents a "typical" base. We restrict our attention to military installations with more than 1 million square feet of building space, which constitute over $96 \%$ of DoD's building footprint worldwide. To provide information that is relevant to the most bases we have created a series of model installations that span common conditions.

\subsection{DoD Installations' Energy Consumption}

The primary metric that characterizes an installation's backup power demand is its peak critical load. This establishes the size of generation needed for a microgrid and is roughly proportional to the number of buildings that have critical loads. On average, electricity accounts for $51 \%$ of all military installation energy consumption (3). The fraction of that electric load that is critical can vary from less than $10 \%$ to over $50 \%$. Based on reviews of dozens of installations, critical load is typically $30 \%$ of total load and hourly peak load is commonly $170 \%$ of the annual average load.

We can estimate the range of critical hourly peak load found across DoD installations based on data in DoD's Annual Energy Management and Resilience Report (3). Figure 12 shows a histogram of the number of installations as a function of the hourly peak critical load under the assumptions described above.

${ }^{11}$ Base Structure Report - Fiscal Year 2018 Baseline

https://www.acq.osd.mil/eie/Downloads/BSI/Base\%20Structure\%20Report\%20FY18.pdf. 


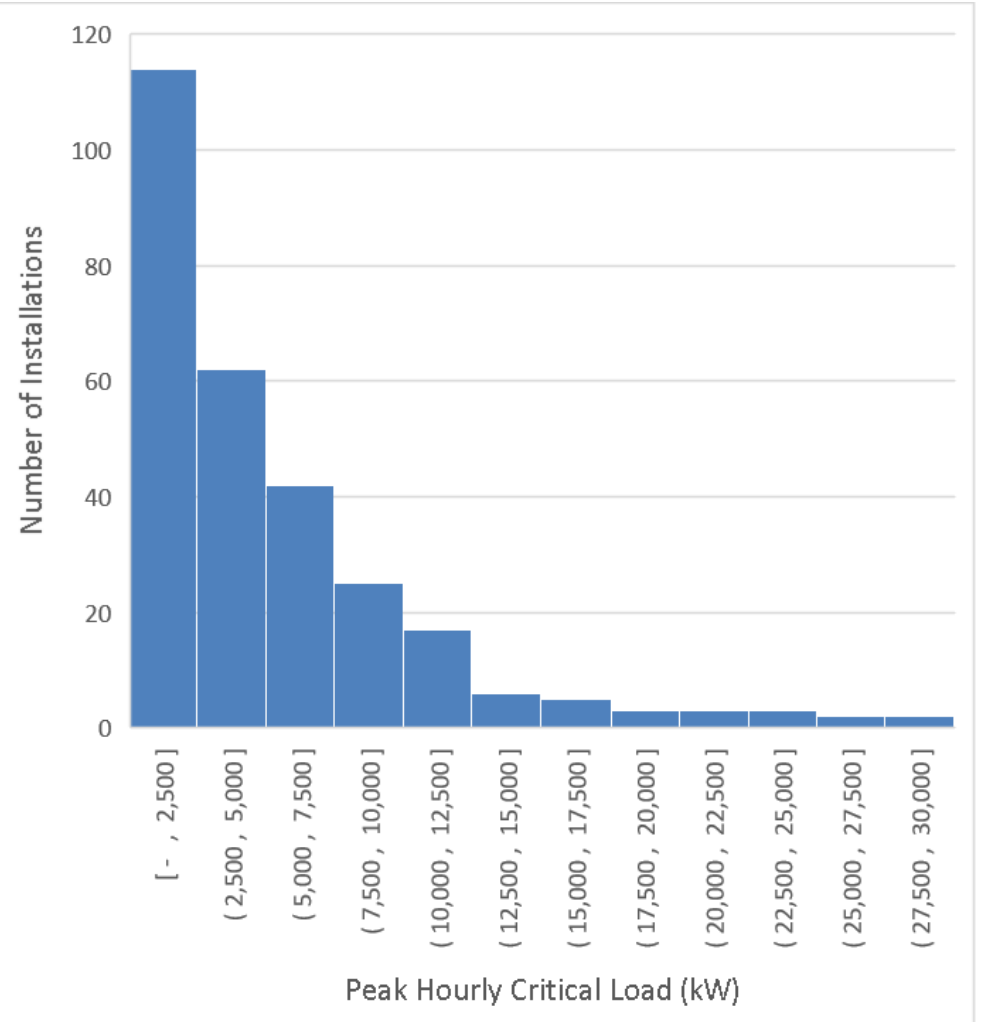

Figure 12. Peak hourly critical load for all DoD installation with buildings with more than 1 million square feet of floorspace

To sample this variation of critical peak load, we have modeled four installation with peak critical electric loads ranging from1 MW to $20 \mathrm{MW}$.

\subsection{Installation Case Studies}

For each installation, we model three potential energy assurance architectures based on EDGs. The first is a system where a single EDG is tied to each building with a critical load. The second is where two EDGs are tied to each building with a high priority critical load. Most military bases use a combination of these two approaches, where most buildings with critical load are supported by a single EDG and subset of buildings with high priority critical loads are supported by two EDGs. We assume that buildings with critical loads have roughly equal energy loads on the order of $100 \mathrm{~kW}$. The number of buildings requiring backup power ranges from 8 for the small base to 160 for the very large base.

The third energy assurance architecture is a microgrid powered by centralized EDGs. We design the microgrid with $\mathrm{N}+1$ EDGs, where $\mathrm{N}$ is the number of EDGs to meet the peak critical load and one additional EDG for higher reliability. To assess the performance of a microgrid system requires knowing the critical load profile. This is important because a network (i.e., a microgrid) of EDGs can support any building with a critical load, and, thus, the effective redundancy of EDGs as shown above varies over time depending on the load profile. For building tied EDGs this information is irrelevant as each EDG cannot support any other building even if it has excess capacity. Hourly load profiles from multiple military installations were gathered and reviewed. Based on similar size bases, typical load profiles were created for each size base being modeled. 
The size of the microgrid EDGs for each modeled base was chosen in accordance with common engineering trade-offs. EDG sizes were constrained to $250 \mathrm{~kW}, 750 \mathrm{~kW}$, and 2,000 kW. Figure 15 shows the annual critical load profiles with the generator step sizes used in this study. Using the smallest size EDG will lead to the largest number of EDGs and therefore the highest O\&M maintenance costs. Using larger EDGs can limit the ability to expand cost effectively to meet future load growth and maintain a common fleet of EDGs.

Based on these considerations, the number of buildings, and the size and number of EDGs for the microgrid configurations for each base are listed in Table 6.

Table 6. Annual Hourly Peak Critical Load, Number of Critical Buildings, and N Generators for the Microgrid Scenario for the Small, Medium, Large, and Very Large Bases Sizes Modeled

\begin{tabular}{|l|c|c|c|c|}
\hline Base & Small & Medium & Large & Very Large \\
\hline $\begin{array}{l}\text { Peak Critical Load } \\
\text { (MW) }\end{array}$ & 1 & 5 & 10 & 20 \\
\hline $\begin{array}{l}\text { Mean Critical } \\
\text { Load (MW) }\end{array}$ & 0.6 & 2.8 & 6.3 & 11.2 \\
\hline $\begin{array}{l}\text { \# Buildings With } \\
\text { Critical Loads }\end{array}$ & 8 & 40 & 80 & 160 \\
\hline $\begin{array}{l}\text { Microgrid EDG } \\
\text { Size (kW) }\end{array}$ & 250 & 750 & 750 & 2000 \\
\hline $\begin{array}{l}\text { Number of } \\
\text { Microgrid EDGs }\end{array}$ & 5 & 8 & 15 & 11 \\
\hline
\end{tabular}

The hourly load profiles in units of kW and EDG capacity is shown in Figure 13.
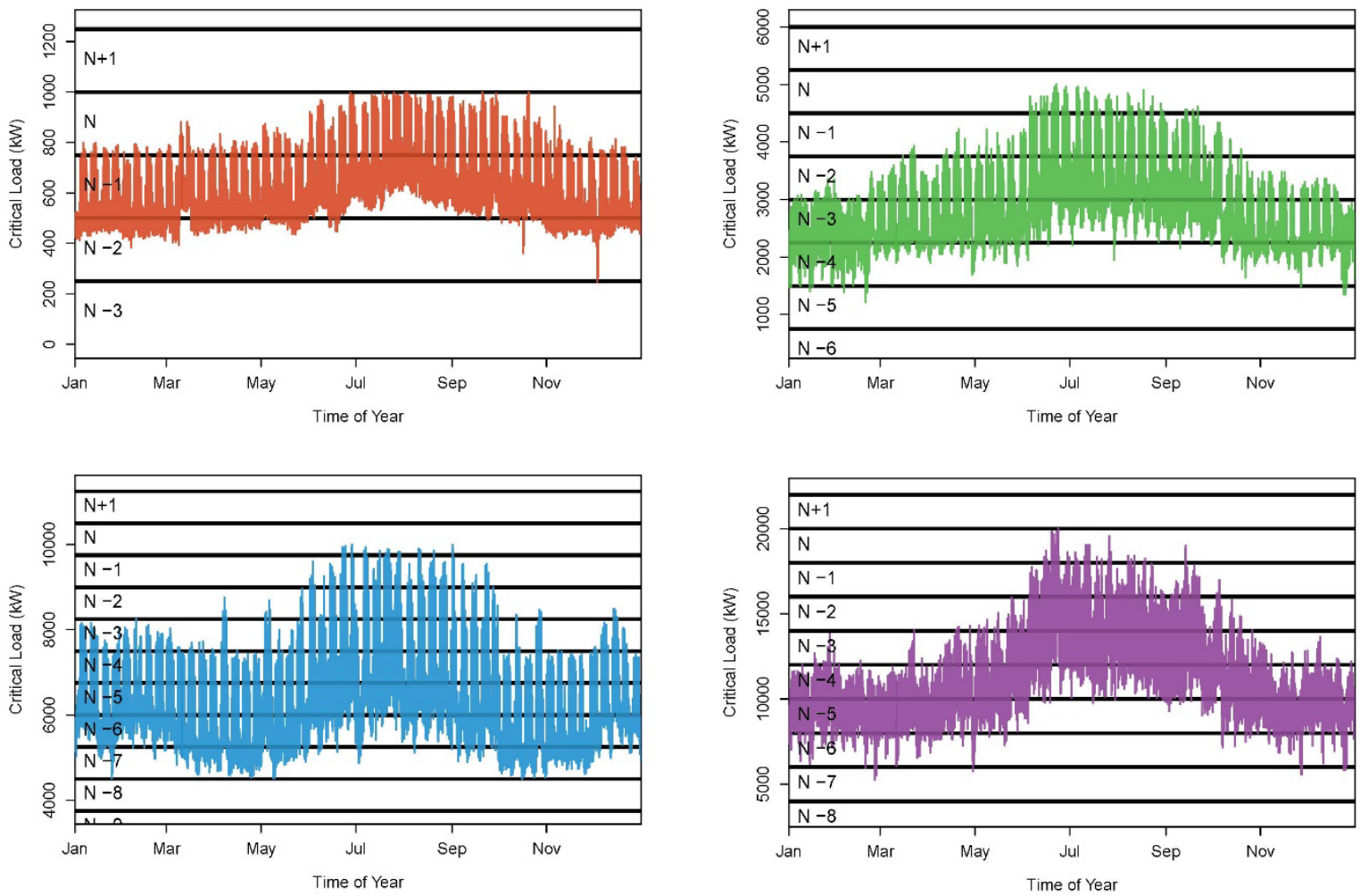

Figure 13. Modeled base critical load profiles with generator step sizes for small (top left), medium (top right), large (bottom left), and very large (bottom right) bases 


\section{Installation Results}

Three reliability system performance metrics are illustrated below for well-maintained EDGs. Poorly maintained generators do not meet the needs of military installations independent of how they are arranged. Even in a microgrid configuration, the loss of multiple generators within a few days due to poor maintenance yields inadequate performance.

First, we show the probability that all critical load will be $100 \%$ supported as function of outage duration for a microgrid versus one EDG tied to each building. This is the most sensitive metric in that any loss of load is considered a failure. Next, we show the mean fraction of lost load for a microgrid and the mean fraction of buildings without power for a single EDG per building tied systems. In the microgrid case, the loss of load can be managed by shedding lower priority critical loads to maintain microgrid stability. In the case of building-tied systems, there is no action that can compensate for the EDG's failure. Finally, we look at the impact on the highest priority critical loads, typically only a fraction of the total critical load. These are loads that support high priority critical missions, which must be sustained. For this case we will compare a microgrid to a system of two EDGs per building.

Probability to Support 100\% of Critical Load: Figure 14 shows the probability of meeting 100\% of the critical load for an $\mathrm{N}+1$ microgrid and a single EDG tied to each building for the four model bases using the mean estimates for a well-maintained individual EDG's reliability. This is a stringent metric and it shows the large difference between a microgrid reliability due to EDG failures and single building-tied EDGs. The larger the base, the more building-tied generators are required, which increases the likelihood that one or more generators will fail. 

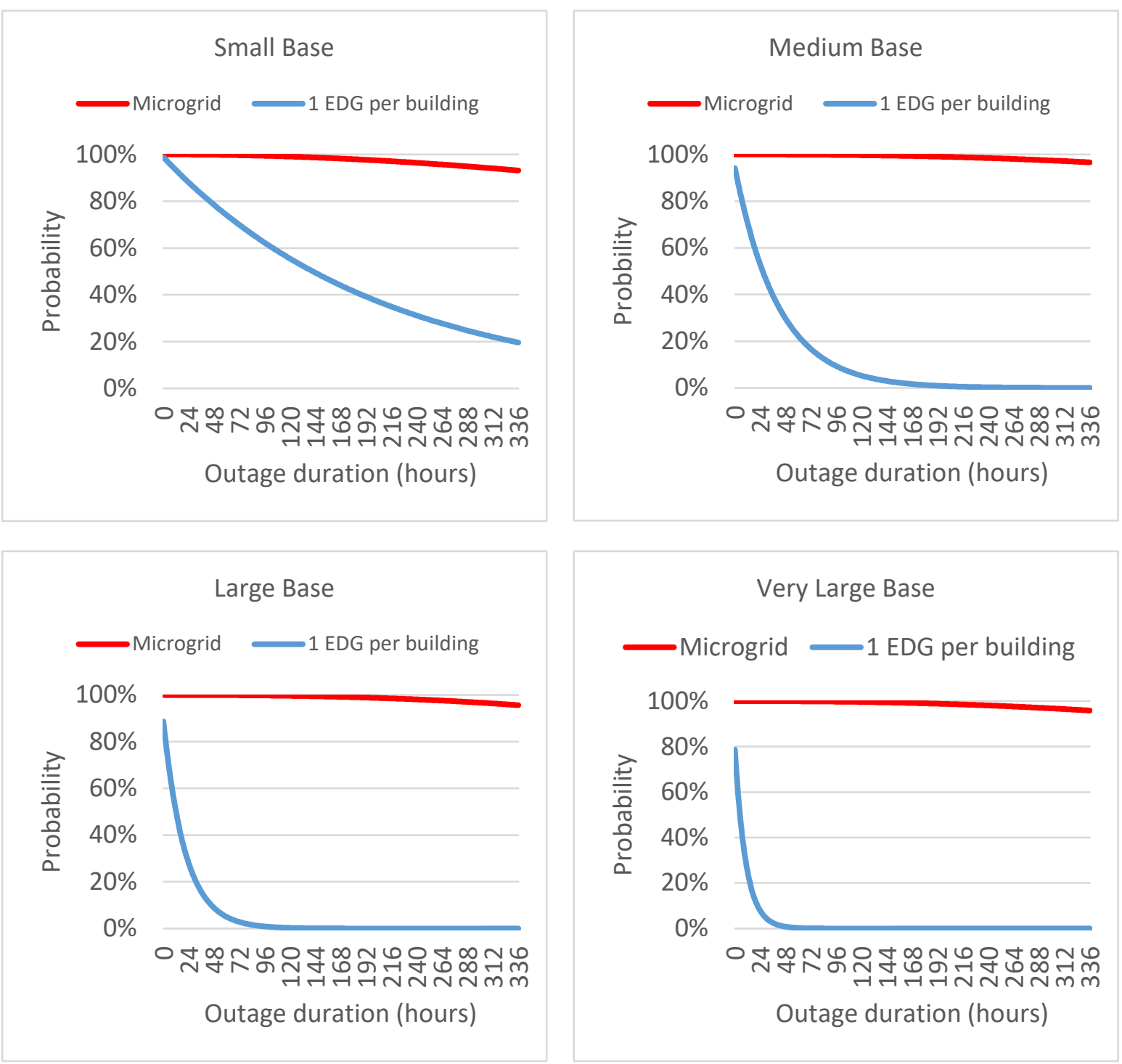

Figure 14. The probability of meeting $100 \%$ of the critical load requirement for outages up to 14 days (336 hours). Small (top left), medium (top right), large (bottom left), and very large (bottom right) bases

Table 7 shows the $90 \%$ confidence range for the performance of the $\mathrm{N}+1$ microgrid configuration and the single stand-alone EDG per building for outages of 1, 3, 7, and 14 days. The confidence range is based on the $90 \%$ confidence intervals for an EDG's reliability data shown in Table 4. The ranges show the uncertainty in the results is much smaller than the large difference between the two architectures. In addition, although the number of EDGs determines the performance of the stand-alone systems, microgrid performance is impacted by both the number of centralized EDGs and the characteristics of the load curve. 
Table 7. $90 \%$ Confidence Ranges for the Probability of Meeting $100 \%$ of the Critical Load for a Microgrid and a Single Stand-Alone Building-Tied EDG at 1, 3, 7, and 14 days

\begin{tabular}{|r|c|c|c|c|c|c|c|c|}
\hline \multicolumn{1}{|c|}{ Base } & \multicolumn{2}{|c|}{ Very Large } & \multicolumn{2}{|c|}{ Large } & \multicolumn{2}{c|}{ Medium } & \multicolumn{2}{c|}{ Small } \\
\hline Architecture & $\begin{array}{c}\text { Microgrid } \\
(\%)\end{array}$ & $\begin{array}{c}\text { Stand- } \\
\text { Alone } \\
(\%)\end{array}$ & $\begin{array}{c}\text { Microgrid } \\
(\%)\end{array}$ & $\begin{array}{c}\text { Stand- } \\
\text { Alone } \\
(\%)\end{array}$ & $\begin{array}{c}\text { Microgrid } \\
(\%)\end{array}$ & $\begin{array}{c}\text { Stand- } \\
\text { Alone } \\
(\%)\end{array}$ & $\begin{array}{c}\text { Microgrid } \\
(\%)\end{array}$ & $\begin{array}{c}\text { Stand- } \\
\text { Alone } \\
(\%)\end{array}$ \\
\hline 1 day & 100 & $3-17$ & 100 & $17-41$ & 100 & $41-64$ & 100 & $84-91$ \\
\hline 3 days & 100 & $0-1$ & 100 & $1-9$ & $99-100$ & $8-29$ & $99-100$ & $60-78$ \\
\hline 7 days & $98-100$ & 0 & $98-100$ & 0 & $95-99$ & $0-6$ & $97-99$ & $32-57$ \\
\hline 14 days & $91-98$ & 0 & $90-98$ & 0 & $85-97$ & 0 & $87-97$ & $10-32$ \\
\hline
\end{tabular}

Expected Lost Critical Load or Buildings: The average expected fraction of lost load for each base for a microgrid and single EDG architecture is shown in Figure 15. This figure highlights how a microgrid is expected even after a two-week outage to be able to meet nearly $100 \%$ of the critical load. The small loss of load can be compensated by the microgrid shedding lower priority critical loads. For EDGs tied to individual buildings, there is no such opportunity. By the end of a two-week outage one should expect to lose the ability to provide electric power to one or two buildings on a small base, while on a very large base, one will lose the ability to provide power to 21 to 40 buildings if single stand-alone EDGs are used. 

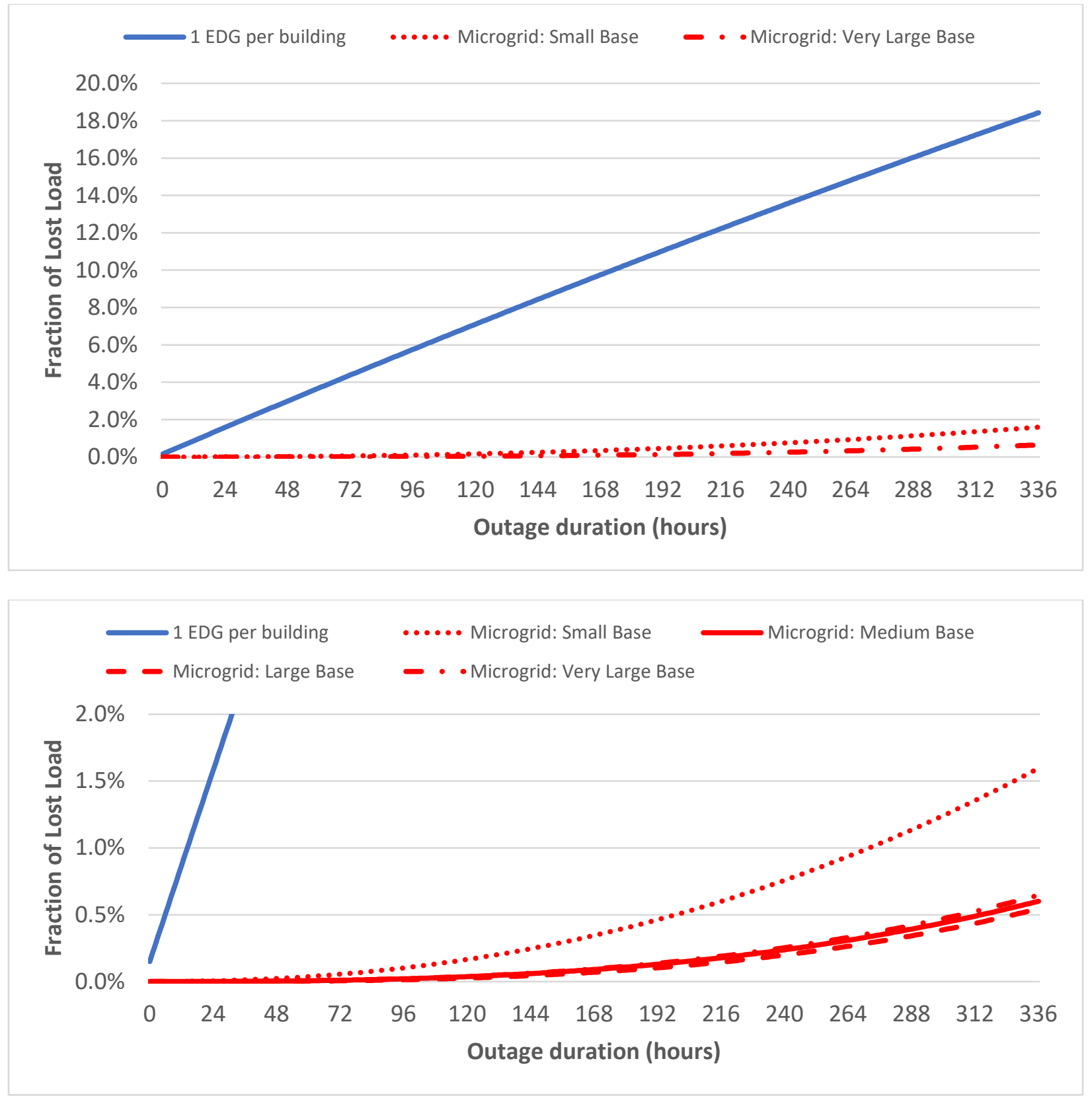

Figure 15. Mean expected lost load for a single EDG per building architecture (blue) and microgrids (red) on different size bases.

The fraction of buildings without power is the same for a stand-alone EDG system for each base. As shown in Section 4, it is independent of the number of buildings. Table 8 translates these results into the expected number of buildings without power for a stand-alone system of single EDGs tied to individual buildings and the load the must be shed for a microgrid to remain operational. Table 8 shows the $90 \%$ confidence range for the performance of the microgrid configuration and the single stand-alone EDG per building for outages of 7 and 14 days. The confidence range is again based on the $90 \%$ confidence intervals for an EDG's reliability shown in Table 8. 
Table 8. $90 \%$ Confidence Ranges for the Load a Microgrid Must Shed and the Number of Buildings with Critical Load That Will Not Have Power if One Uses a Single Stand-Alone Building-Tied EDG at 7 and 14 Days

\begin{tabular}{|l|c|c|c|c|c|c|c|c|c|c|}
\hline Base & \multicolumn{2}{l|}{ Very Large } & \multicolumn{2}{l|}{ Large } & \multicolumn{2}{l|}{ Small } \\
\hline Outage Duration & 7 days & 14 days & 7 days & 14 days & 7 days & 14 days & 7 days & 14 days \\
\hline \multicolumn{7}{|c|}{ Stand-Alone } & & & & \\
\hline $\begin{array}{l}\text { Fraction of Lost } \\
\text { Load (\%) }\end{array}$ & $7-13$ & $13-25$ & $7-13$ & $13-25$ & $7-13$ & $13-25$ & $7-13$ & $13-25$ \\
\hline $\begin{array}{l}\text { Number of } \\
\text { Buildings without } \\
\text { Power }\end{array}$ & $11-21$ & $21-40$ & $6-11$ & $11-20$ & $3-5$ & $5-10$ & 1 & $1-2$ \\
\hline & & & & & & & & \\
\hline $\begin{array}{l}\text { Fraction of Lost } \\
\text { Load (\%) }\end{array}$ & $<0.2$ & $0.2-2$ & $<0.2$ & $0.2-2$ & $<0.2$ & $0.2-2$ & $<0.7$ & $0.7-3$ \\
\hline $\begin{array}{l}\text { Average kWs Not } \\
\text { Supported }\end{array}$ & $4-26$ & $26-$ & $2-11$ & $11-97$ & $1-7$ & $6-43$ & $1-5$ & $4-21$ \\
\hline
\end{tabular}

Probability to Meet Highest Priority Critical Loads: Perhaps the most important metric is the impact of EDG reliability on the ability to provide power to the highest priority critical loads on a base. Often, the highest priority missions require critical loads be supported across different buildings to be operational. A microgrid has an advantage in that it can prioritize loads in real time, ensuring that the highest priority loads are always satisfied. Stand-alone systems cannot change priority or shift DERs during an outage. The only way they can increase reliability for high priority critical loads is by increasing the number of EDGs linked to any individual building. Figure 16 shows the probability of meeting the highest priority load for situations where the high priority load is $10 \%$ and $25 \%$ of the total critical load for a microgrid and two stand-alone EDG per buildings. 


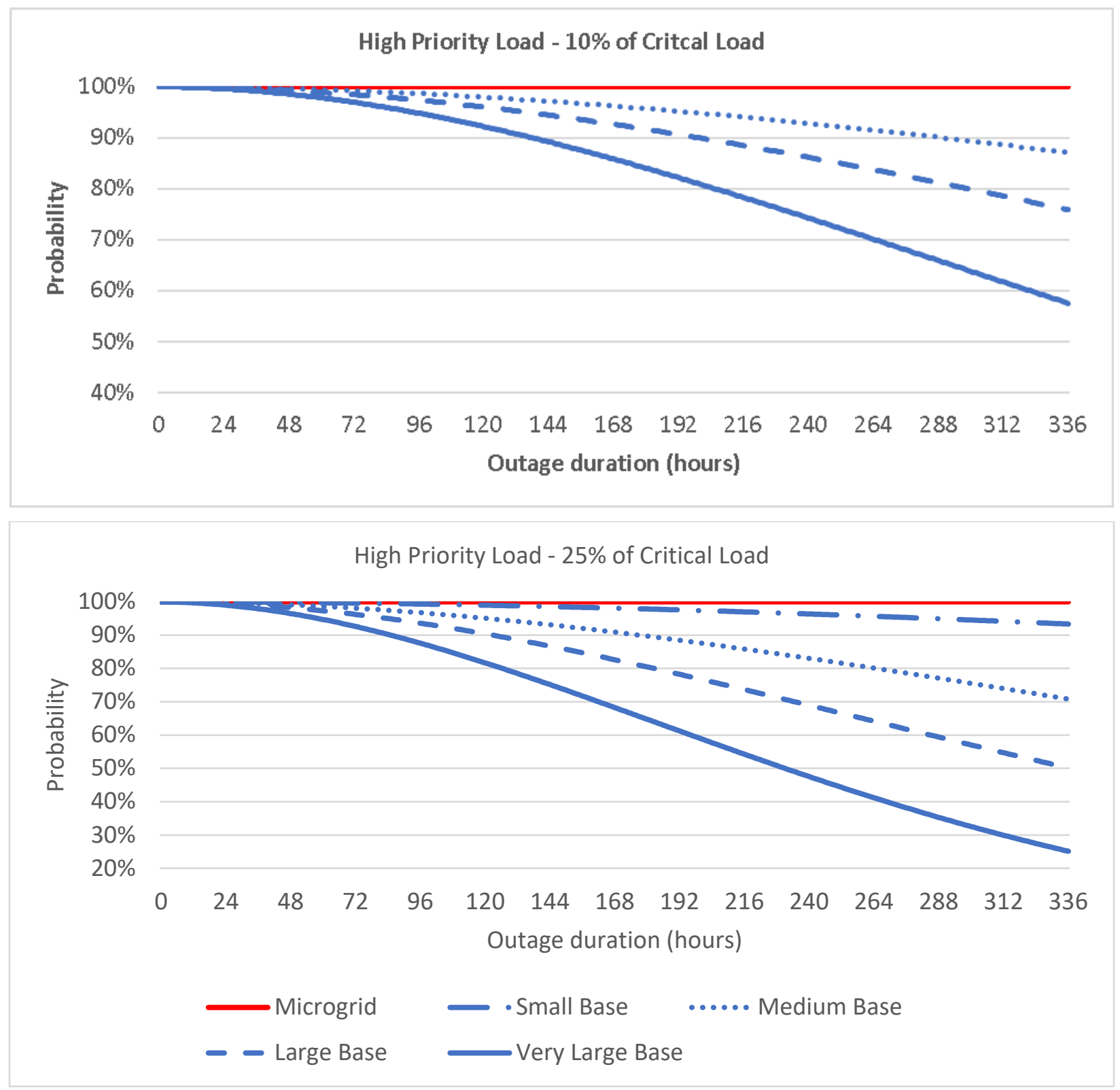

Figure 16. Probability of meeting $10 \%$ and $25 \%$ of highest priority critical load for small, medium, large, and very large bases. The red curve represents the results for all bases supported by a microgrid. The blue results are for bases where two EDGs are deployed for every building containing a high priority critical load.

For the small base no results are shown for the top $10 \%$ priority because $10 \%$ is less than one building.

Microgrids have close to a $100 \%$ probability of having power generation capacity sufficient to always meet the highest priority critical load. Stand-alone systems with two EDGs per building do not come close to meeting DoD's needs at medium, large, and very large bases. By two weeks into an outage, the probability based on mean reliability metrics drops to $25 \%$ to $71 \%$ for the top $25 \%$ priority critical load and from $58 \%$ to $87 \%$ for the top $10 \%$ priority critical load. For small bases, $25 \%$ of the critical load is contained in only two buildings. Even in this case, by two 
weeks into an outage the probability based on mean reliability metrics drops to $93 \%$ as comparted to near a $100 \%$ for a microgrid. Table 9 illustrates the range of probabilities that $10 \%$ and $25 \%$ of critical loads will be powered based on the $90 \%$ confidence interval for the individual EDG reliabilities.

Table 9. $90 \%$ Confidence Ranges for the Probability That the 10\% and $25 \%$ Highest Priority Buildings With Critical Loads Will Have Power When Using a Stand-Alone System of Two EDGs Per Building for outage durations of 7 and 14 Days

\begin{tabular}{|c|c|c|c|c|c|c|c|c|}
\hline Base & \multicolumn{2}{|c|}{ Very Large } & \multicolumn{2}{|c|}{ Large } & \multicolumn{2}{c|}{ Medium } & \multicolumn{2}{c|}{ Small } \\
\hline $\begin{array}{c}\text { \% of Critical } \\
\text { Load }\end{array}$ & $10 \%$ & $25 \%$ & $10 \%$ & $25 \%$ & $10 \%$ & $25 \%$ & $10 \%$ & $25 \%$ \\
\hline 7 Days & $75 \%-$ & $48 \%-$ & $86 \%-$ & $69 \%-$ & $93 \%-$ & $83 \%-$ & NA & $96 \%-$ \\
& $93 \%$ & $83 \%$ & $96 \%$ & $96 \%$ & $98 \%$ & $95 \%$ & & $99 \%$ \\
\hline 14 Days & $36 \%-$ & $8 \%-50 \%$ & $60 \%-$ & $28 \%-$ & $77 \%-$ & $53 \%-$ & NA & $88 \%-$ \\
& $76 \%$ & & $87 \%$ & $71 \%$ & $93 \%$ & $84 \%$ & & $97 \%$ \\
\hline
\end{tabular}

The numbers should be compared to the expected $100 \%$ available power from a microgrid for the entire $90 \%$ confidence interval for reliability metrics. We cannot assume that even a system of two EDGs per building will be able to provide sufficient power for the high priority critical loads in the event of a multiweek outage.

How many EDGs tied to each building are required to have a high confidence that the highest priority load will all have power? Figure 17 illustrates that for a large base one would require at least four EDGs per building to have a greater than $99 \%$ probability that power is available for the highest $10 \%$ of priority critical loads.

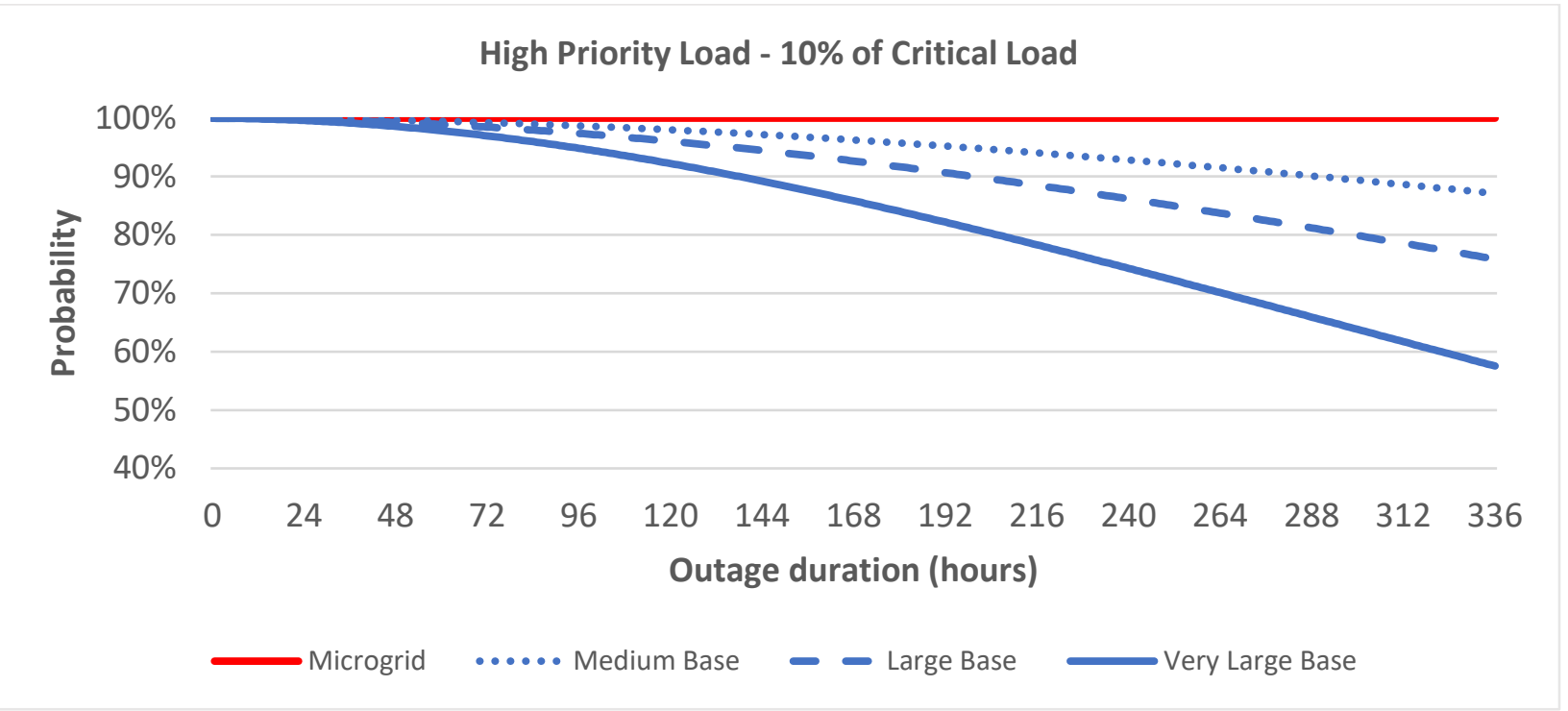

Figure 17. Probability of meeting $10 \%$ of highest priority critical load for a large base as a function of the number of stand-alone EDG per building as compared to a microgrid 
All three metrics provide overwhelming evidence that stand-alone building-tied EDG systems cannot provide the level of confidence DoD needs for power to be available to support critical missions. Microgrid configurations provide a robust source of power for critical loads due to their network configuration. But microgrids do introduce other vulnerabilities that must be managed. These include cyber vulnerabilities and dependence on the on-base distribution system. Cyber vulnerabilities can be mitigated by appropriate cyber defenses. On-base distribution systems reliability varies dramatically from currently being the primary cause of outages to never being the cause. Proper maintenance and well-known mitigations can significantly reduce the likelihood of the on-base distribution system's vulnerabilities. 


\section{Conclusions}

The current default solution for backup energy at military installations relies on EDGs. This is most often accomplished by either a single stand-alone generator or two generators tied to an individual building with critical loads. Less common, but with increasing frequency, diesel generators are networked and serve as the primary DER for a microgrid. EDGs can be unavailable due to maintenance, failure to start and carry the load, or failure to run during a grid outage. System-level reliability ${ }^{12}$ is a key performance criterion that should be considered when assessing the relative value of different backup energy system for a specific installation. In the absence of such information, military installations cannot quantitively assess their current energy assurance vulnerabilities nor evaluate alternative approaches. This work has analyzed the impact of EDG reliability on base backup energy systems.

There has been an absence of realistic estimates for the reliability of individual emergency diesel generators. Using IEEE reported mean time between failure results in incorrect predictions. New estimates for the reliability of modern commercial emergency diesel generators that are commonly used on DoD installations based on empirical data sets are provided. Poorly maintained emergency diesel generators are unlikely to run more than a few days, and well-maintained emergency diesel generators have only an $80 \%$ likelihood of being operational at the end of a two-week outage.

For a military installation, where multiple buildings house critical loads, the impact of the reliability of a well-maintained EDG is significant. Installations that rely on a single stand-alone EDGs tied to individual buildings with critical loads are unlikely to have power for all these loads over a two-week outage. It is likely that a small base will lose power to a few buildings while larger bases will lose power to dozens of buildings. A microgrid, which is composed of a network of centralized EDGs, has a high probability that all buildings with critical loads can be supported throughout a two-week outage. The expected microgrid lost load is very small and can be managed by shedding lower priority loads. Of greatest concern is power for the highest priority critical loads. Stand-alone building-tied EDG systems manage this by placing two EDG per building (a backup to the backup). Although this improves the likelihood of having power, it does not provide the level of reliability DoD needs. Such stand-alone systems will have less than a 50\% probability of supporting the highest priority critical loads for a two-week grid outage on larger bases. Microgrid systems can prioritize loads in real time and essentially can guarantee power availability for the highest priory critical load.

EDGs must be well-maintained if they are to be relied on for providing power for more than a few hours. If backup power is required for multiple days, stand-alone building-tied EDGs cannot be relied on by themselves to provide backup power for critical loads. Diesel generator based microgrid configurations provide a robust source of power for critical loads due to their network configuration. But microgrid do introduce other vulnerabilities that must be managed. These include cyber vulnerabilities and dependence on the on-campus distribution system. The analysis presented here also does not consider hybrid microgrid systems that combine EDGs with intermittent renewable energy and storage. In future work we will report on the reliability provided by intermittent renewable energy coupled to battery storage and the impact of the reliability of the on-base distribution system.

\footnotetext{
${ }^{12}$ System reliability is a measure of the likelihood that the critical loads will be supported for a required duration during a grid outage.
} 


\section{References}

1. Marqusee, Jeffrey, Craig Schultz, and Dorothy Robyn. Power Begins at Home:Assured Energy for U.S. Military Bases. 2017.

https://www.pewtrusts.org/ /media/assets/2017/01/ce_power_begins_at_home_assured_energy_ for_us_military_bases.pdf.

2. U.S. Department of Defense. National Defense Strategy. Washington, D.C.: U.S. Department of Defense. 2018.

3. - 2018 Annual Energy Management Report. Washington, D.C.: U.S. Department of Defense. 2018.

4. Larsen, Peter, Kristina LaCommare, Joseph Eto, and James Sweeney. Assessing Changes in the Reliability of the U.S. Electric Power System, LBNL - 188741. Berkeley, CA: Lawrence Berkeley National Laboratory. 2015.

5. U.S. Department of Energy. Multiyear Plan for Energy Cybersecurity. Washington, D.C: U.S. Department of Energy. 2018.

6. Marqusee, Jeffrey, and Don Jenket. "Reliability of Emergency and Standby Diesel Generators: Impact on Energy Resiliency Solutions." 2020. Applied Energy, Vol. 268. 15 June 2020. 114918.

7. Birolini, Alessandro. Reliability Engineering Theory and Practice. New York: Springer, 2007.

8. 493, IEEE. Design of Reliable Commercial Power Systems. 2007.

9. 3000.6, IEEE Standard. Recommended Practice for Anayzing Reliability Data For Equipment Used in Industrial and Commercial Power Systems. 2018.

10. U.S. Department of the Army. TM 5-698-5, Survey of Reliability and Availability Information for Power Distribution, Power Generation, and Heating, Ventilating and Air Conditioning (HVAC) Components for Commercial, Industrial and Utility Installations. July 2006.

11. Du, Y., J. Burnett, and S.M. Chan. "Reliability of Standby Generators in Hong Kong Buildings." IEEE Transactions on Industry Applications 39 no. 6 (2003).

12. Thompson, Christopher C., Peyton S. Hale, Jr., and Robert G. Arno. "Decanting the Data: The Gold Book Presents Equipment Reliability Refreshment." IEEE Transactions on Industry Application 482 (2012).

13. Schroeder, John A. Enhanced Component Performance Study: Emergency Diesel Generators 1998-2016. INL/LTD 17-44204. April 2018.

14. Fehr, Stephen John. Emergency Diesel-Electric Generator Set Maintenance and Test Periodicity. Ph.D. diss., Old Dominion University. DOI: 10.25777/q2nk-n411

https://digitalcommons.odu.edu/emse_et, 2017. 
15. National Fire Protection Association. NFPA 110 Standard for Emergency and Standby Power Systems. 2019.

16. U.S. Department of Defense. Unified Fcility Criteria (UFC): OPERATION AND MAINTENANCE (O\&M): GENERATORS. Washington, D.C.: U.S. Department of Defense. 2018. UFC 3-540-07.

17. U.S. Nuclear Regulatory Commission. "Reactor Operational Experience Results and Databases." Last modified OCtober 1, 2019. https://nrcoe.inl.gov/resultsdb/. 